bioRxiv preprint doi: https://doi.org/10.1101/2021.05.13.443994 · this version posted May 14,2021 . The copyright holder for this preprint (which was not certified by peer review) is the author/funder, who has granted bioRxiv a license to display the preprint in perpetuity. It is made available under aCC-BY-NC-ND 4.0 International license.

\title{
Partial response electromyography as a marker of the individual stopping latency
}

\author{
Liisa Raud ${ }^{1,2}$, Christina Thunberg ${ }^{2,3}$, René J. Huster ${ }^{2,3}$
}

1 Center for Lifespan Changes in Brain and Cognition, Department of Psychology, University of Oslo, Norway

2 Cognitive and Translational Neuroscience Cluster, Department of Psychology, University of Oslo, Norway

3 Multimodal Imaging and Cognitive Control Lab, Department of Psychology, University of Oslo, Norway

Corresponding author: Liisa Raud, liisa.raud@psykologi.uio.no 


\begin{abstract}
Response inhibition is among the core constructs of cognitive control. It is notoriously difficult to quantify from overt behavior, since the outcome of successful inhibition is the lack of a behavioral response. Therefore, model-based approaches have been developed to estimate the inhibition latency, such as the stop signal reaction time (SSRT) using the stop signal task. However, the model assumptions underlying the SSRT can be difficult to meet in practice. Recently, partial response electromyography (prEMG) has been introduced as a physiological measure to capture individual stopping latencies. PrEMG refers to muscle activity initiated by the go signal that plummets after the stop signal, before its accumulation to a full response. We provide a comprehensive overview of prEMG in a stop signal task, together with practical tips for data collection and analysis. Our analysis indicates that prEMG is a unique and reliable measure of stopping and we encourage its widespread use to investigate response inhibition.
\end{abstract}




\section{Introduction}

Response inhibition, or the ability to stop actions, is a core construct of cognitive control and is widely studied among cognitive and clinical neuroscientists. Most of our knowledge about inhibitory capability is derived from a single behavioral measure, the stop signal reaction time (SSRT), which quantifies the time the stopping process needs to countermand an already initiated action. SSRTs are slower in patients with attention deficit/hyperactivity disorder, obsessive compulsive disorder, drug abuse, schizophrenia, Parkinson's disease, and Tourette syndrome among others (Jahanshahi et al., 2015; Lipszyc and Schachar, 2010; Smith et al., 2014; Snyder et al., 2015; Wylie et al., 2013; Yaniv et al., 2017). However, accumulating evidence suggests that SSRT estimations may be unreliable in many practical settings (Bissett et al., 2021b; Bissett and Logan, 2014; Matzke et al., 2017; Skippen et al., 2019; Verbruggen et al., 2013). Here, we scrutinize electromyography (EMG) recordings from the muscles that are successfully stopped as a potential physiological marker to capture individual differences in stopping.

The stop signal task is among the most common experimental tasks used to study inhibition, with more than 7000 publications using the task in the last years (Verbruggen et al., 2019). The principle of the task is very simple: participants react repeatedly to a go signal with a button press, but need to inhibit this response when a stop signal appears after the go signal with a variable delay (stop signal delay; SSD). The resulting go reaction time distribution, probability of responding despite stop signal presentation, and the resulting SSDs are the necessary variables for calculating the SSRT.

The SSRT estimation is based on the independent race model (or horse race model), in which go and stop processes independently race against each other, and whichever process wins this race, determines whether a planned action will be executed or inhibited (Band et al., 2003; Logan and Cowan, 1984). The theoretical basis of the model is well established and an extension of this model also has biological plausibility (Boucher et al., 2007). However, recent work indicates that SSRT estimates may be inaccurate in many practical cases, for example due to extensive slowing of go responses (Verbruggen et al., 2013), violations of the independence assumptions at short SSDs (Bissett et al., 2021b), or failures to initiate the stop process (Skippen et al., 2019). The available solutions focus on the derivation of putatively improved SSRT estimates, either by refining the estimation method (Verbruggen et al., 2019), discarding participants or trials that show obvious violations of the model assumptions (Bissett et al., 2021b, 2021a), or by proposing alternative modelling approaches (Heathcote et al., 2019; Matzke et al., 2017, 2013). However, the field has been in a remarkable standstill when it comes to developing alternatives to the SSRT that could potentially bypass the issues related to model assumptions in the first place. 
A notable exception is the proposition to derive a more direct measure of the stopping latency from EMG. Specifically, EMG recorded at the effector muscles that generate the response can be used to capture the time-point when the motor activity begins to decline in the muscles. In a number of successfully stopped trials (trials without a registered response), surface EMG recordings still capture small bursts of muscle activity. These bursts are presumably responses initiated after the go signal that are stopped before the muscle activity culminates in a button press. We have termed these responses partial response EMG (prEMG; see Box 1 for visualization and reflections on the naming). The proposition to use the latency of the prEMG as an indicator of an individual's stopping speed seems to have originated independently in two different labs (Atsma et al., 2018; Raud and Huster, 2017). Raud and Huster used surface EMG from thumb muscles in a selective stop signal task and measured the prEMG peak latency to be about $150 \mathrm{~ms}$, which was significantly shorter than the corresponding SSRT of about $200 \mathrm{~ms}$. Atsma and colleagues used intramuscular recordings of the upper-limb muscle during reaching movements. They reported a prEMG offset latency (the time-point when muscle activity returned to the baseline level) at $165 \mathrm{~ms}$, compared with an SSRT of $244 \mathrm{~ms}$. While their methods and goals were different, both studies showed that the EMG-derived stopping latency was much faster than that of the model-based SSRT.

While using the prEMG latency as an index of stopping capability is a relatively recent suggestion, recording EMG in a stop signal task is not particularly novel by itself. Earlier work identified that muscle activity can be stopped at any time before the movement is completed, and additionally focused on the time-courses of different electrophysiological measures, i.e. EMG, electrocardiography (ECG), and electroencephalography (EEG; De Jong et al., 1990; Jennings et al., 1992; van Boxtel et al., 2001). These studies identified the motor cortex as a site of inhibition, yet they also showed that the peripheral nervous system is affected as well. Several further studies attempted to interpret the prEMG within the context of the horse race model. For instance, trials with prEMG seem to roughly correspond to the middle part of the go-RT distribution, indicating that these trials have a relatively fast go process that nonetheless is slow enough to be suppressed by the stop process (De Jong et al., 1990; van Boxtel et al., 2001). Furthermore, the occurrence of the prEMG is dependent on the SSD, as prEMG frequency declines and its amplitude increases with increasing SSD (Coxon et al., 2006). Interestingly, EMG in unsuccessful stop trials may be affected by inhibition processes as well, as these trials exhibit lower EMG amplitudes, but prolonged motor times (the time between EMG onset and the registered response) compared with standard go responses (van de Laar et al., 2014). This violates the assumption of stochastic independence underlying the independent horse race model, as unsuccessful stop trials are supposedly the same as fast go responses and should therefore be unaffected by the stop signal. The dependence of go and stop processes was recently directly shown 
by Raud et al., (2020a) who found a positive correlation between go-locked prEMG onsets and stoplocked prEMG peak latencies at the level of single stop trials.

The earlier timing of the prEMG peak latency compared to the SSRT has been replicated in a number of independent studies (Hannah et al., 2020; Jana et al., 2020; Raud et al., 2020a, 2020b; Sundby et al., 2020; Tatz et al., 2021; Thunberg et al., 2020). However, while the existence of the prEMG is relatively well-established, its characteristics and utility has not been explored sufficiently to declare it an unambiguous biomarker of the stopping latency. We therefore present a thorough investigation of the prEMG in the stop signal task. Specifically, we benchmark the PrEMG peak latency against the SSRT and the existing horse race model framework; investigate potential relationships between response onset, stopping, and the SSD at a single trial level; present novel information about stopping variability; and estimate the reliability of the EMG measures. We additionally provide practical tips for data collection and analysis, accompanied by publicly available analysis code that can easily be adjusted to new datasets. With that, we demonstrate the full potential of prEMG with the aim to encourage and accelerate future studies to adopt it as a standard outcome measure of inhibition tasks.

\section{Box 1. Definition and naming of partial response EMG}

Partial response EMG (prEMG) refers to small muscle bursts in successful stop trials (third panel in the figure below). These bursts are elicited in response to the go signal but stopped in response to the stop signal before the muscle activation culminates in a button press. As such, the trial itself is considered a successful stop trial, but the prEMG onset and peak latencies indicate the exact timing of the go- and stop processes at the effector muscles, respectively.
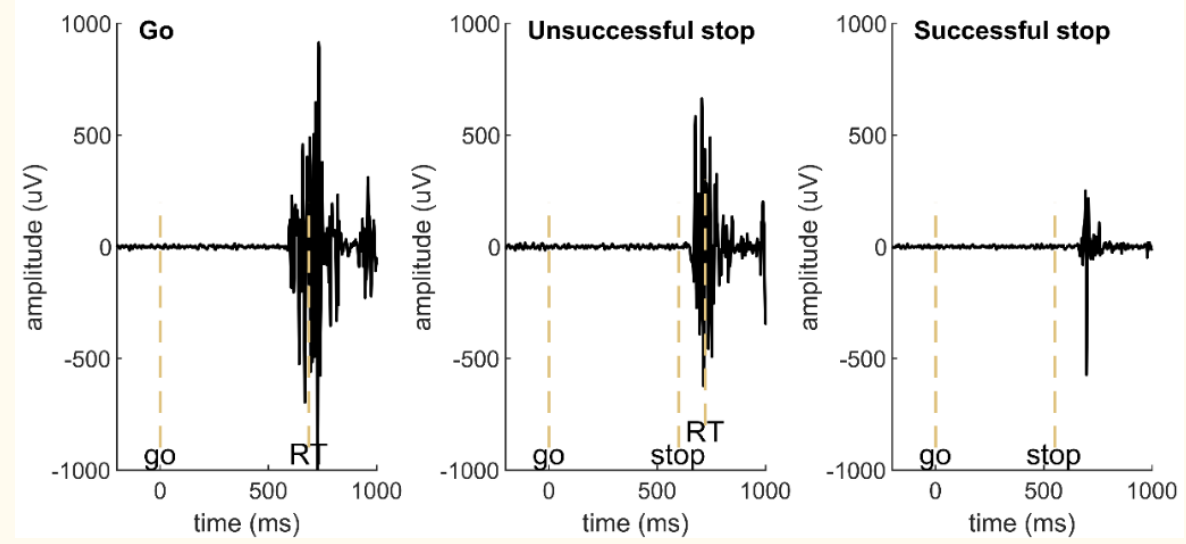

Examples of EMG signals in a go, unsuccessful stop, and successful stop trial in an example participant. EMG in go and unsuccessful stop trials is associated with a button press (marked by RT), while no button press is registered in successful stop trials and the muscle burst therefore qualifies as prEMG. The dashed vertical lines mark the onset of go signal, stop signal, and the recorded reaction time (RT).

Whereas we use the term prEMG here, this term has not been used consistently across studies. However, we believe prEMG to describe the phenomenon most accurately without any 
assumptions about the underlying mechanisms. Below, we list the terms found in the previous literature.

Partial EMG. Early papers used this term and it is a more concise version of the term prEMG. However, partial EMG implies that the EMG signal itself is incomplete, which is not the case. Rather, the EMG signal in successful stop trials itself is perfectly valid but different from other EMG bursts because the response is not completed.

Interrupted EMG. McGarry and Franks $(2003,1997)$ used this term for muscle bursts in stop trials with onset slopes similar to those of the go EMG, which indicates that the stop process exerted its effects after the go process had activated the motor pool. This was contrasted with partial response $E M G$ with gentler onset slopes, assumed to reflect a stop process interfering prior to motor pool activation, thus resulting in a weakened activation (or a leakage signal). However, they used a stop signal task with unusually short SSDs from -100 to $100 \mathrm{~ms}$ relative to the go signal, and it is unclear whether these responses can be dissociated in a more typical stop signal task with SSDs $>100 \mathrm{~ms}$.

Partial error. This term is consistently used in tasks in which an erroneous automatic response needs to be overridden by a correct response (e.g. Burle et al., 2002; Coles et al., 1995; Rochet et al., 2014). In such tasks, the term 'error' is appropriate. However, the prEMG in the stop signal task is not erroneous as it is elicited by the go signal, which is perfectly in line with the task instructions.

Subthreshold EMG. This term was used by Raud and Huster (2017), indicating that the EMG in successful stop trials did not reach the threshold to trigger the button press. However, such thresholds are relatively arbitrary and depend on the physical properties of the response device. In addition, this naming becomes inappropriate if continuous movements are of interest, as for example is the case with measurements of force or movement trajectories.

Cancel time. This term, suggested by Jana et al. (2020), specifically refers to the prEMG peak latency. Whereas appealing due to its simplicity, it is potentially misleading as it implies a direct cancellation mechanism for stopped movements. Yet, alternative mechanisms may cause the same EMG phenomenon, such as reciprocal inhibition due to the activation of antagonist muscle groups, or a simple reduction in response maintenance. 


\section{Results}

\section{Behavior and EMG in a stop signal task}

We analyzed surface EMG recorded over the thumb muscles (abductor pollicis brevis) from 46 healthy young adults who performed a standard stop signal task. The primary task was a choice reaction time task where participants had to respond to a go signal either with a left or right hand button press. In $24 \%$ of the trials, a stop signal occurred instructing the participants to stop their response. The behavioral results (Table 1) were in line with previous studies, with stopping accuracies at $50 \%$ and an SSRT (integration method with RT replacement for go omissions) of $199 \mathrm{~ms}$. We also estimated behavioral parameters using Bayesian Ex-Gaussian Estimation of Stop Signal Distribution approach accounting for go and trigger failures (BEESTS; Matzke et al., 2019, 2013), which resulted in a SSRT of $202 \mathrm{~ms}$ (Table 1).

\begin{tabular}{lrrrrr} 
& mean & sd & median & min & max \\
\hline BEHAVIOR & & & & & \\
go accuracy (\%) & 97.92 & 2.21 & 98.76 & 91.23 & 100.00 \\
go errors (\%) & 0.69 & 0.88 & 0.37 & 0.00 & 5.12 \\
go omissions (\%) & 1.40 & 1.95 & 0.73 & 0.00 & 8.04 \\
go RT (ms) & 545.28 & 77.11 & 549.04 & 398.29 & 715.24 \\
SSD (ms) & 347.50 & 97.59 & 349.53 & 155.71 & 551.60 \\
SSRT (ms) & 198.99 & 29.63 & 202.00 & 120.05 & 263.97 \\
stop accuracy (\%) & 49.88 & 1.84 & 50.46 & 43.06 & 54.17 \\
unsuccessful stop RT (ms) & 480.47 & 70.85 & 496.64 & 351.11 & 671.92 \\
& & & & & \\
BEESTS PARAMETERS & & & & & \\
\hline go failures (\%) & 1.56 & 2.18 & 0.76 & 0.04 & 9.12 \\
trigger failures (\%) & 1.27 & 1.62 & 0.51 & 0.28 & 8.94 \\
go RT (mean; ms) & 546.51 & 78.83 & 548.71 & 396.93 & 722.25 \\
go RT (sd; ms) & 113.52 & 28.97 & 112.25 & 65.57 & 167.46 \\
SSRT (mean; ms) & 201.83 & 21.39 & 200.03 & 166.22 & 248.85 \\
SSRT (sd; ms) & 38.25 & 52.89 & 27.91 & 14.94 & 378.03 \\
\hline
\end{tabular}

Table 1. Behavioral and BEESTS parameters summary statistics. RT - reaction time; sd - standard deviation; SSD - stop signal delay; SSRT - stop signal reaction time. $\mathrm{N}=46$.

EMG bursts were identified automatically using a threshold-based algorithm (see Box 2 and Materials and Methods for details). EMG bursts were detected in $99 \%$ of go trials and $98 \%$ of unsuccessful stop trials. Note that while $100 \%$ detection rate is expected in those trials, variations in signal quality 
(increased baseline muscle tone, unrelated movement artifacts etc.) led to slight conservative bias in the detection algorithm. PrEMG was detected in $23 \%$ of successful stop trials.

Averaged EMG waveforms for each trial type are shown in Figure 1 and the summary statistics are presented in Table 2. When time-locked to go signal onset, the trials with a registered button press showed large EMG activity (Figure 1A). Note that go-locked averaging over varied SSDs causes a smeared waveform for prEMG. We used Bayesian hypothesis testing to compare the EMG onset latencies and peak amplitudes between trial types. This confirmed that the EMG onsets were earlier in unsuccessful stop trials than in go trials $\left(\mathrm{BF}=1.32 * 10^{24}\right)$ and that the prEMG onset latencies were similar to the EMG onset latencies in go trials $(B F=0.28)$. When time-locked to the EMG onset (Figure 1B), the rising flanks of the EMG waveforms were remarkably similar across all trials but reached very different amplitudes. The peak amplitudes in successful stop trials were considerably smaller than in go $\left(B F=2.77 * 10^{29}\right)$ and unsuccessful stop trials $\left(B F=1.91 * 10^{27}\right)$. Interestingly, EMG amplitudes in unsuccessful stop trials were also smaller than those in go trials $\left(B F=2.32 * 10^{7}\right)$, suggesting that the former were affected by stop signal processing despite the registered button press.

\begin{tabular}{llll} 
variable & go & $\begin{array}{l}\text { successful } \\
\text { stop }\end{array}$ & $\begin{array}{l}\text { unsuccessful } \\
\text { stop }\end{array}$ \\
\hline count & $655(25)$ & $23(13)$ & $106(5)$ \\
percentage & $99(2)$ & $23(14)$ & $98(2)$ \\
$\begin{array}{l}\text { onset latency (ms) } \\
\text { peak latency* (ms) }\end{array}$ & $487(81)$ & $481(91)$ & $422(74)$ \\
$\begin{array}{l}\text { peak amplitude } \\
\text { (a.u.) }\end{array}$ & $12(1)$ & $4(1)$ & $11(1)$ \\
$\begin{array}{l}\text { motor time (ms) } \\
\text { rise time (ms) }\end{array}$ & $57(13)$ & NA (NA) & $58(14)$ \\
\hline
\end{tabular}

Table 2. Means (and standard deviations in brackets) of the EMG variables. Motor time refers to the difference between the EMG onset latency and the reaction time. Rise time refers to the difference between EMG onset and peak latency. ${ }^{*}$ peak latencies are time-locked to the stop signal onset. $\mathrm{N}=46$.

When time-locked to stop signal onset, a very clear prEMG waveform appeared (Figure 1C), which started declining at $165 \mathrm{~ms}$ after the stop signal. A similar decline also occurred in unsuccessful stop trials, although they peaked slightly earlier at $99 \mathrm{~ms}$ after the stop signal $\left(\mathrm{BF}=3.47 * 10^{12}\right.$ ). Speculatively, the steep decline of the falling flank seen in unsuccessful stop trials may suggest that unsuccessful stop trials were also affected by the processes triggered by the stop signal around the same time as the prEMG decline in successful stop trials. 

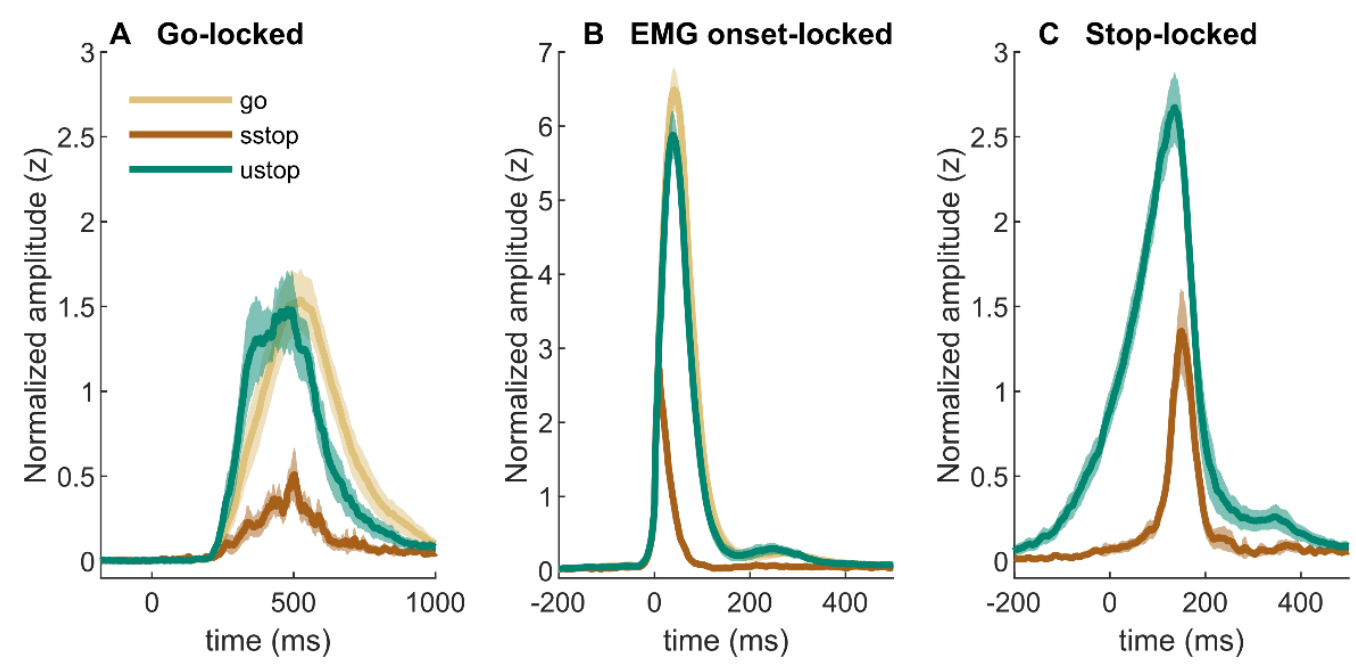

Figure 1. EMG time-courses, time-locked to (A) go signal onset, (B) EMG onset, and (C) stop signal onset. The lines depict averaged activity and the shaded areas depict $95 \%$ confidence intervals across participants. Sstop successful stop; ustop - unsuccessful stop.

We additionally quantified the motor time, i.e. the difference between the EMG onset and the RT, and the rise time, i.e. the difference between the EMG onset and peak latency. Even though the differences are numerically small (Table 2 ), the motor time was longer in unsuccessful stop trials than in go trials ( $B F=5.94$; replicating the finding of van de Laar et al., 2014), but the rise time was shorter in unsuccessful stop trials than in go trials $\left.B F=1.16^{*} 10^{6}\right)$. At only $19 \mathrm{~ms}$, the rise time was very short for the prEMG, indicating that its decline started almost immediately after its onset. This suggests that trials with prEMG may be drawn from a relatively limited pool within the full reaction time distribution. Assuming a stable latency of the stopping process, faster go reactions would have longer rise times. But they also have a higher likelihood to result in unsuccessful stop trials, and thus are likely underrepresented in the sample of prEMG trials. Slower go reactions, on the other hand, are likely to be suppressed even before the onset of muscle activity. 


\section{Box 2. Detecting prEMG and extracting peak latency}

\section{EMG detection}

The identification of EMG bursts is a signal detection problem. Given that the signal-to-noise ratio of EMG data is relatively high, simple threshold-based detection algorithms perform reasonably well. Basic preprocessing steps to further increase the signal-to-noise ratio may include high-pass filtering, rectification or a root mean square transformation, smoothing or low-pass filtering, baseline normalization, and the definition of a temporal window eligible for burst detection. We additionally normalize the full EMG time-courses to be able to keep the detection threshold constant across participants. Our processing pipeline is freely available at the Open Science framework (OSF; Raud et al., 2021). Alternatively, one may refer to the toolbox provided by Jackson and Greenhouse (2019).

Simple threshold-based algorithms may, however, show declined performance in the presence of high baseline EMG activity or when cross-talk from different muscle groups is prevalent. In such cases, burst detection in the frequency domain may outperform detection in the time-domain (see Atsma et al., 2018; Liu et al., 2015 for examples).

\section{Extracting peak latency}

There are several ways to extract the prEMG peak latency. Here, we extracted the peak latency of an EMG burst from each trial in which prEMG was detected, and then averaged across trials to get a summary measure for each participant. This method typically requires extra cleaning, discarding trials with elevated baseline muscle tone or irrelevant muscle artifacts outside of the expected response window. A more robust method is to first average the EMG activity time-point-wise across all successful stop trials with prEMG and then extract a single peak latency from the averaged waveform. A third alternative is to extract the average peak latency from trials stemming from the most frequent SSD (the mode). This has the closest resemblance to the SSRT estimation, (as that is typically calculated at a single SSD, such as the mean SSD), but this method also has significantly fewer available trials, reducing the overall signal-to-noise ratio.

We compared the three methods and found a good correspondence between them (Spearman $r$ between 0.69 and 0.83 ). All three methods also correlated with the SSRT. Peak latency estimates extracted from averaged waveforms appear more normally distributed than estimates from single trials, indicating that the former may represent the more robust estimation approach. However, as this method gives only a single summary measure per participant, it is not appropriate if single trial analyses are of interest. The prEMG peak latency calculated using only the trials at mode SSD seems to be susceptible to outliers, likely because very few trials are used to derive the summary measure. 


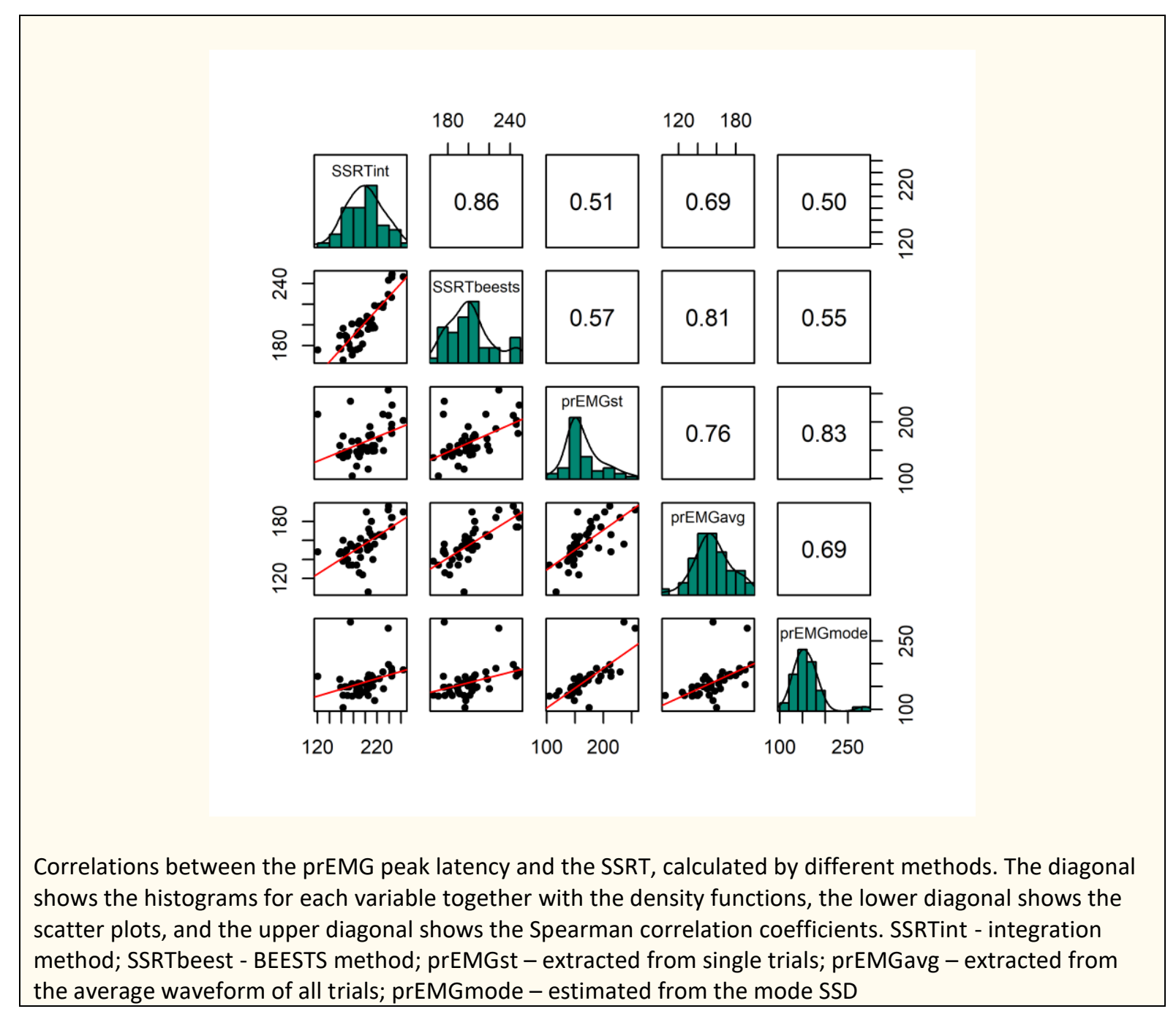

\section{Comparison of prEMG peak latency and SSRT}

There are several different ways to calculate the SSRT. The two state-of-the art techniques are the estimation using the integration method with replacement of go omissions (Verbruggen et al., 2019), and the Bayesian parametric approach (Heathcote et al., 2019; Matzke et al., 2013), which has been extended to incorporate go errors and failures to trigger both the go and the stop process (Matzke et al., 2019, 2017). Where some studies show prevalent trigger failures and considerably faster BEESTSSSRTs than integrations-SSRTs (Skippen et al., 2020, 2019), others find a lower incidence of trigger failures and correspondingly little change in SSRT estimates (Jana et al., 2020). In line with the latter, we found the different SSRT estimates to be comparable in this dataset (BF $=0.34$ ). Both were considerably slower than the prEMG peak latency (integration $\mathrm{BF}=9.6 * 10^{5}, \mathrm{BEESTS} \mathrm{BF}=2.6^{*} 10^{9}$ ). Both SSRTs correlated moderately with the prEMG peak latency (integration $r=0.51$; BEESTS $r=0.57$ for prEMG latency derived from single trials, but see Box 2 for differences in correlations with different prEMG peak latency estimations). Consistent with previous studies, the delay between the prEMG and 
the SSRT (integration) was $33 \mathrm{~ms}(\mathrm{sd}=33)$. This did not correlate with the prEMG detection frequency $(r=0.04)$.

\section{Comparison of behavior- and EMG-based inhibition functions}

Having established the basic summary characteristics and their (non-)correspondence with the SSRT, we set out to investigate how the prEMG relates to the framework of the horse race model of going and stopping. A feature of the stop signal task is that the SSD varies, most commonly due to an online tracking algorithm that adapts the SSDs to each participant's performance. The purpose of this is to capture the SSD where participants manage to stop their response for about $50 \%$ of the time. A key assumption of this approach is that the probability of unsuccessful stopping ( $p$ (button press $\mid$ stop $)$ ) is low (approaching zero) at short SSDs, increases steeply at mid-SSDs, and approaches one at long SSDs. With EMG, this would correspond to the probability of producing EMG at any given stop trial (i.e. $\mathrm{p}(\mathrm{EMG} \mid$ stop$))$, as this implicates that the go-process was at least initiated before stopping process. This probability thus includes all stop trials with detectable EMG, and is conceptually the sum of unsuccessful stop trials and successful stop trials with prEMG. Thus, by definition, the function of $p(E M G \mid s t o p)$ should be shifted to the left from the function of $p$ (button press|stop). In addition, assuming that the successful stop trials with prEMG are drawn uniformly from the distribution of successful stop trials, we would expect the two functions to run in parallel. However, preliminary evidence suggests that prEMG occurs more often at short SSDs (Coxon et al., 2006; De Jong et al., 1990). Given that $p(E M G \mid$ stop) represents the sum of unsuccessful stop trials and successful stop trials with prEMG, this would lead to larger differences between the two functions at short than in long SSDs.

The empirical inhibition functions are depicted on Figure 2. To capture the expected non-linear shape of the functions, we fit a generalized additive mixed model (GAMM), predicting the probability of responding ( $p$ (button press $\mid$ stop) or producing EMG ( $(E M G \mid$ stop)) despite a stop signal as a function of SSD; this model thus also allows for the assessment of the interaction of response type (button, $E M G)$ and SSD (model $\left.R^{2}=0.64\right)$. The effect of SSD was significant $\left(d f=3.18, F=213.91, p<2 * 10^{-16}\right)$, as was the parametric effect of response type $\left(b=0.07, t=9.52, p<2^{*} 10^{-16}\right)$. Further, the interaction term was significant $(\mathrm{df}=1.00, \mathrm{~F}=6.795, \mathrm{p}=0.009)$, with model fits indicating that the difference between detecting a button compared to an EMG response was larger at smaller SSDs. Since $p(E M G \mid$ stop) contains $p$ (button press|stop) as well successful stop trials with prEMG, these effects indicate that prEMG was more common in trials with short SSDs. 


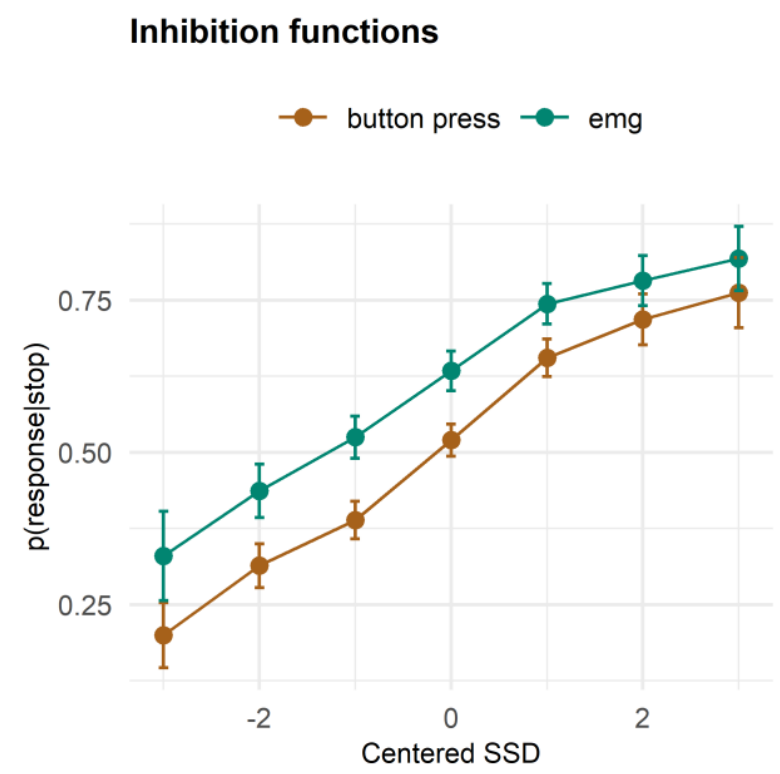

Figure 2. Inhibition functions. Error bars represent 95\% confidence intervals. Note that the error bars are calculated across all trials and participants, ignoring that each participant has several data points.

\section{Stochastic independence of going and stopping}

A key assumption of the horse race model is that the go and stop processes are independent of each other. Consequently, the distribution of the RTs in go trials can be used as a proxy for the unobserved RT distribution in stop trials. Dividing the go-RT distribution at the percentile corresponding to the $p$ (button press $\mid$ stop, SSD) gives estimates of the distribution of trials for a given SSD in which the go process would and would not escape the stop process. Specifically, the portion at the left of this division are the fast go trials that would escape inhibition, and the portion on the right are slow go trials that would be stopped. Deriving an additional division line using the percentile corresponding to p(EMG|stop, SSD), De Jong et al. (1990) extended this framework using the distribution of EMG onset latencies in go trials instead of the RT distribution. They concluded that prEMG was sampled from the distribution just to the right of the division line produced by $p$ (button press/stop, SSD), thus corresponding roughly to the middle part of the go EMG onset distribution (see Figure 3 for an example). However, De Jong's analysis was done on six participants only, and we will therefore extend this analysis here on a much larger sample.

Figure 3 explains some key expectations. Adhering to the independent horse race model, we expected EMG onsets in unsuccessful stop trials to match fast go-EMG onsets. Then, as the $p$ (EMG|stop) is larger than $p$ (button press/stop), there is a second division line derived from the percentile corresponding to $p(E M G \mid s t o p)$, which is shifted to the right. The trials between those two lines should correspond to successful stop trials with prEMG. Consequently, the mean go-EMG onset latencies 
from trials between these two division lines should be equal to the prEMG onsets. Lastly, the go-EMG onsets to the right of the second dividing line should be slower than the prEMG onsets.

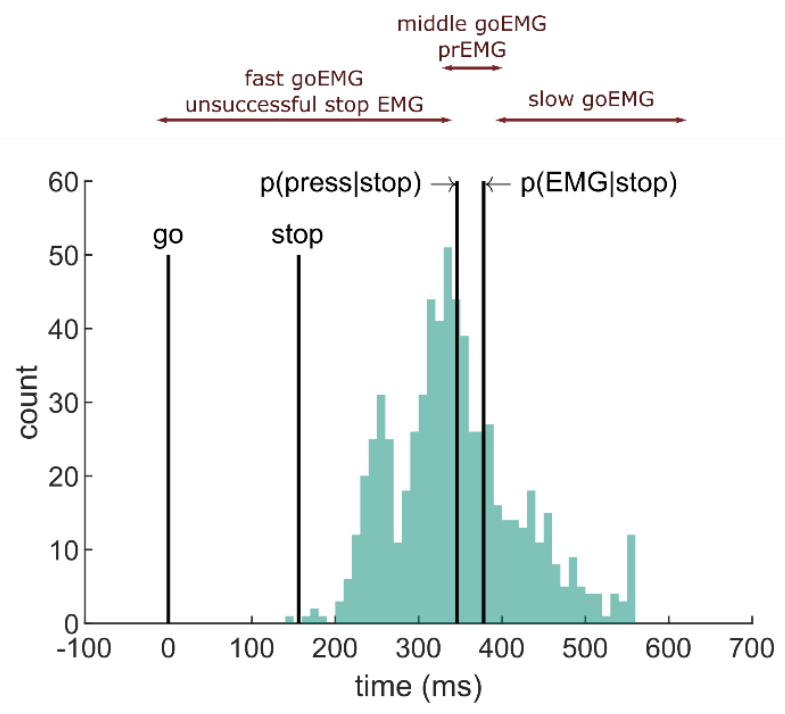

Figure 3. Depiction of the extension of the horse race model on EMG data extracted from an example participant. The histogram depicts the distribution of EMG onsets in go trials, and the vertical lines mark (from left to right) the go signal onset, stop signal onset (mean SSD), percentile corresponding to the probability of producing a response and to the probability of producing EMG in stop trials. Assuming stochastic independence of go and stop trials, unsuccessful stop trials should correspond to the fast go trials (left side of the distribution between go signal and $p$ (press|stop), while prEMG should correspond to the middle partition of the go distribution in between the $p$ (press $\mid$ stop) and $p(E M G \mid$ stop).

The EMG onset latencies, averaged over all trials within each partition of the go-EMG distribution, are listed in Table 3. The data indicate a deviation from the independence assumption already for the left side of the distribution, as the average EMG onset in unsuccessful stop trials was slower than the average EMG onset in fast go trials $\left(B F=2.67 * 10^{9}\right)$. Secondly, the average prEMG onsets were faster than the corresponding average onset of the middle partition of the go-EMG distribution (BF = $2.17^{*} 10^{4}$ ), which contrasts the prediction that these should be roughly equivalent. Lastly, the slow goEMG onsets occurred later than the prEMG onsets, as expected $\left(B F=2.81 * 10^{21}\right)$. As go-EMG distributions are skewed and the means of each portion may misrepresent the central values, this analysis was repeated using median values instead, but the pattern of the results remained the same. Thus, our results indicate that extending the horse race model to the EMG is not applicable and, consequently, estimating the SSRT based on go-EMG distributions should be avoided. Note that the RTs of unsuccessful stop trial do not always correspond to the fast go-RT portion either (van de Laar et al., 2014). This issue may thus be relevant at the RT level as well. 


\begin{tabular}{lrrrrr} 
& mean & sd & median & min & max \\
\hline goEMG_fast & 401 & 67 & 410 & 260 & 555 \\
goEMG_medium & 506 & 80 & 500 & 347 & 678 \\
goEMG_slow & 608 & 108 & 582 & 416 & 844 \\
usEMG & 422 & 74 & 430 & 272 & 596 \\
prEMG & 481 & 91 & 474 & 290 & 701 \\
\hline
\end{tabular}

Table 3. EMG onsets in go trials divided into fast (between go onset and p(press/stop), medium (between $\mathrm{p}($ press $\mid$ stop $)$ and $\mathrm{p}(\mathrm{EMG} \mid$ stop $))$ and slow (>p(EMG|stop)) trials. usEMG = unsuccessful stop EMG; prEMG = partial response EMG (successful stop trials)

\section{prEMG characteristics as a function of SSD.}

Even though the patterns of EMG onsets do not seem to be in accordance with assumptions of the horse race model, it is important to determine the prEMG characteristics across all SSDs to establish whether stopping estimates based on the prEMG are representative of the overall stopping behavior. Based on previous findings, we expected the prEMG frequency to decline and its amplitude to increase with increasing SSD. Further, as go RTs tend to correlate positively with the SSDs, we expected that the prEMG onset latencies (calculated relative to the go signal) would increase with SSDs as well. Note that the prEMG peak latency, in contrast, is calculated relative to the stop signal and thus reflects the speed of the stop process. Critically, it has never been asked whether the prEMG peak latency remains stable at different SSDs.

We fit four GAMMs to predict the prEMG probability, onsets, amplitudes, and peak latencies from the SSDs (Figure 4). As expected, prEMG probability decreased with the SSD ( $d f=1.92, F=24.28, p=$ $2.47 * 10^{-10}, R^{2}=0.09$ ). Similarly, we found increased EMG onsets with increasing SSD (df $=2.60, F=$ $\left.420.7, p<2.00 * 10^{-16}, R^{2}=0.36\right)$. The prEMG peak latencies decreased with SSD ( $d f=2.29, F=11.77, p$ $\left.=9.71^{*} 10^{-6}, R^{2}=0.06\right)$, indicating that stopping may be slower at short SSDs. PrEMG amplitudes increased slightly with longer SSDs, with the smooth term at the border of significance $(\mathrm{df}=1.66, \mathrm{~F}=$ $\left.4.16, p=0.056, R^{2}=0.02\right)$. 

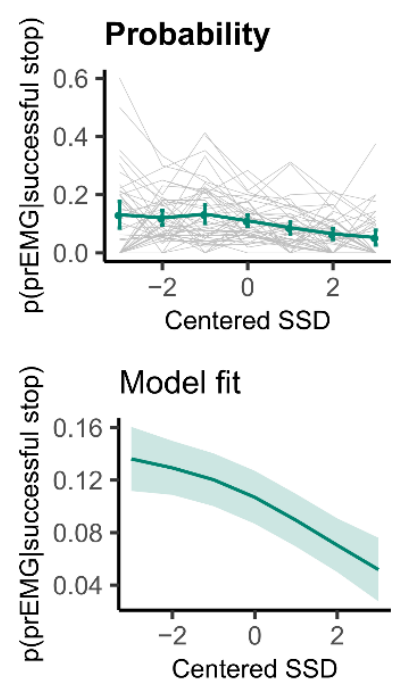

Onset latency

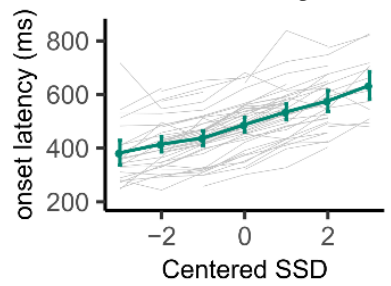

Model fit

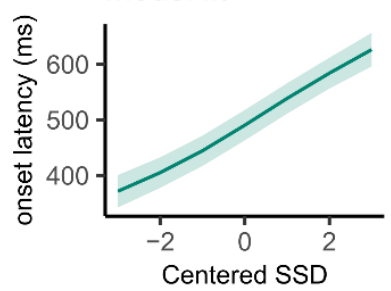

Peak latency

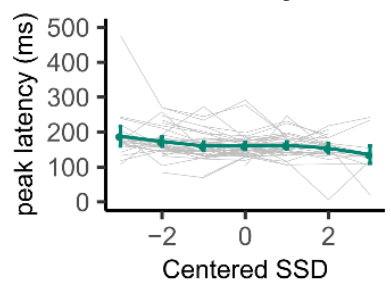

Model fit

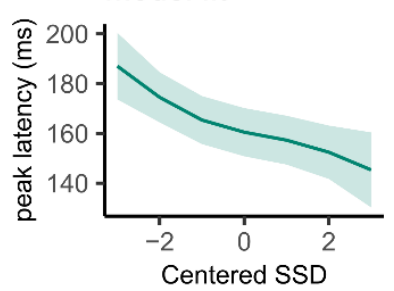

Peak amplitude

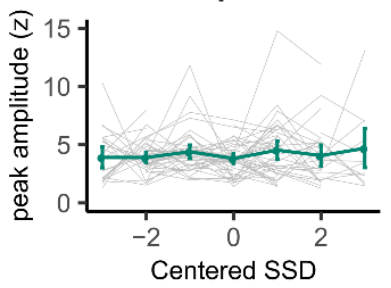

Model fit

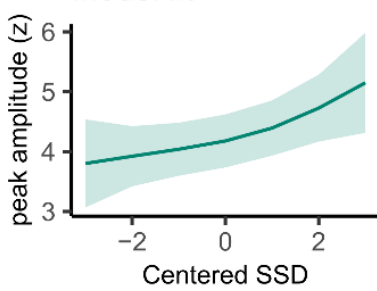

Figure 4. prEMG probability, onset latency, peak latency, and peak amplitude. The upper panels represent empirical data across all participants. Green lines represent the group average with $95 \%$ confidence intervals and grey lines represent each participant. The lower panels depict the model fit of the generalized additive models with $95 \%$ confidence intervals. Note the different $y$-axes between empirical data and model fits.

\section{prEMG peak latency as a function of time on task.}

Another important question is whether prEMG remains stable across the experiment. It is possible that inhibition becomes automatic over time and loses its cognitive control element (Best et al., 2016; Verbruggen et al., 2014; Verbruggen and Logan, 2008). Consequently, prEMG peak latency may decrease after practice. We tested this by fitting a GAMM model predicting prEMG peak latency by trial order while controlling for SSD (model $\mathrm{R}^{2}=0.04$ ), but we found no evidence that the trial order would affect prEMG peak latency ( $d f=1.173, F=0.11, p=0.843)$. We also tested whether the trial order affected prEMG frequency by predicting the existence of prEMG in a given trial from the trial order using logistic regression (while controlling for the SSD), but this model yielded no trial order effects either $(b=-0.03, z=-0.92, p=0.357)$. Note that these results do not exclude that stopping may be automatic to begin with nor that automatism affects underlying processing (Raud et al., 2020b; Verbruggen et al., 2014), but simply indicates that stopping performance did not change with practice in a single session.

\section{Single trial associations of prEMG onset latency, peak latency, and area under the curve}

A clear advantage of the prEMG is that it allows to study the dynamics of going and stopping at a single trial level. A simplified set of predictions outlining different dependencies between going and stopping are depicted in Figure 5, based on prEMG onset latencies (go-locked), peak latencies (stop-locked), 
and areas under the curves (AUC) from onset to peak. The AUC integrates the latency and amplitude of each prEMG burst and was calculated as the cumulative sum of point-wise amplitudes from onset to peak latency. Note that these scenarios are constructed assuming stable SSDs. This simplification helps to delineate the expected outcomes for different a-priori hypotheses regarding the dependencies between going and stopping at the single trial level.
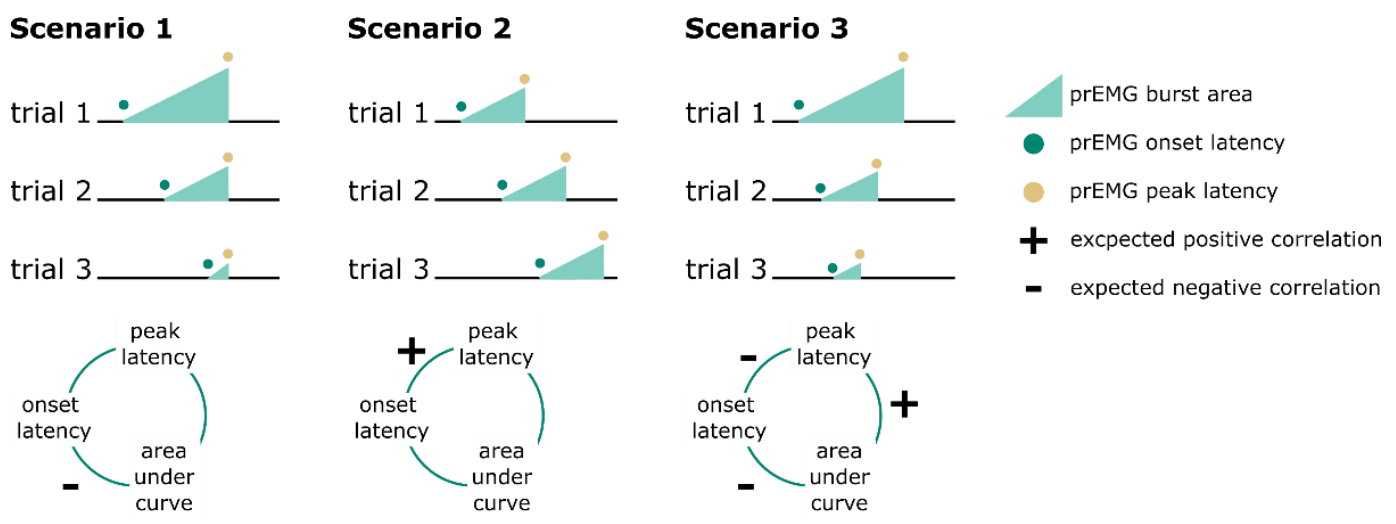

Figure 5. Predicted directions of single trials correlations between EMG onset latency, peak latency, and area under the curve (calculated as the cumulative sum of point-wise amplitudes from onset to peak latency) under three different scenarios.

Scenario one represents the independent horse race model, where there is considerable variability of the go process, but a constant and independent stop process. In this case, earlier prEMG onset leads to a larger AUC, but peak latencies are stable and independent of the onsets and AUCs.

Scenario two represents a case where delayed prEMG onset is associated with delayed prEMG peak latencies (as reported in Raud et al., 2020a), resulting in stable AUCs. This could be due to instances like competing resources for go and stop processes or attentional lapses that delay overall processing.

Scenario 3 represents a different dependency between the go and stop processes, in which more potent motor responses (i.e. earlier onsets and larger AUC) are associated with delayed stopping. This would result in a negative relationship between prEMG onset and peak latencies and between the onset latencies and AUCs, but a positive relationship between the peak latencies and the AUCs.

We tested these scenarios by fitting GAMMs predicting a) peak latency from onset latency, b) peak latency from AUC, c) onset latency from AUC. Onset latencies and SSDs correlated highly at the single trial level $(r=0.88)$. To account for this, we predicted onsets from the SSDs for each participant and used the residuals instead of original onset values. AUCs were log-transformed since the original values were extremely skewed towards low values. 
The data showed evidence for scenario 2 (Figure 6) with a strong positive relationship between onset and peak latencies (edf $=3.47, F=1957, p<2 * 10^{-16}, R^{2}=0.72$ ). There was also a small negative association between onset latencies and the AUCs (edf $=1.00, F=29.25, p=7.93^{*} 10^{-8}, R^{2}=0.03$ ), but no relationship between peak latencies and AUCs (edf $=1.00, F=0.09, p=0.77, R^{2}<0.001$ ). As such, there was strong evidence for dependencies between going and stopping at the single trial level, where delayed going was associated with delayed stopping. Note that none of these relationships were significant at the group level (i.e. taking the mean for each variable for each participant and fitting the same models), thus these results apply for single trial data only.

A Onset and peak

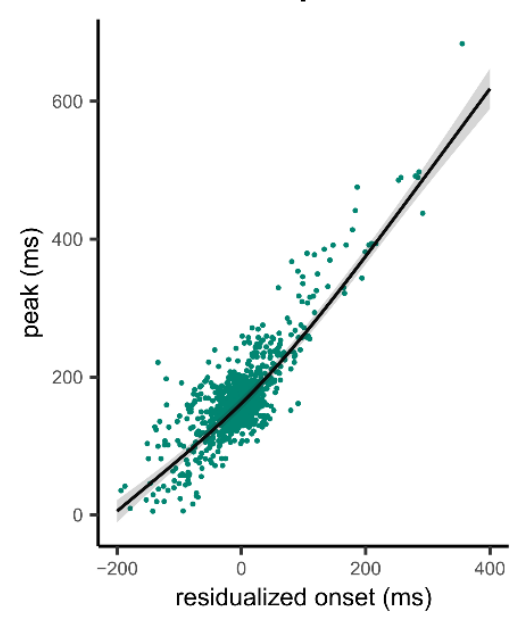

B Peak and AUC

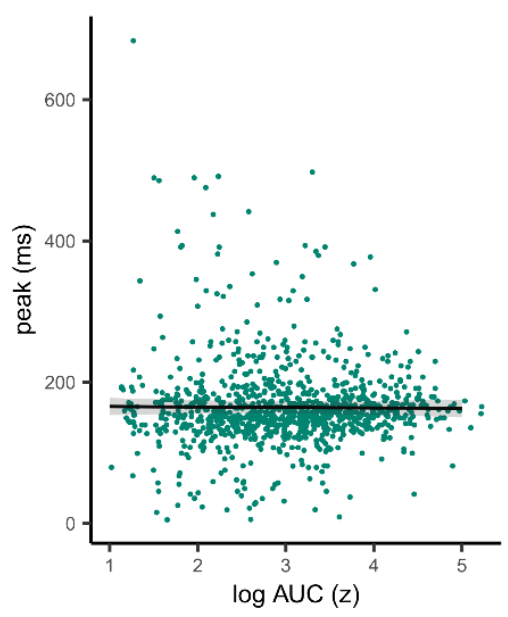

C Onset and AUC

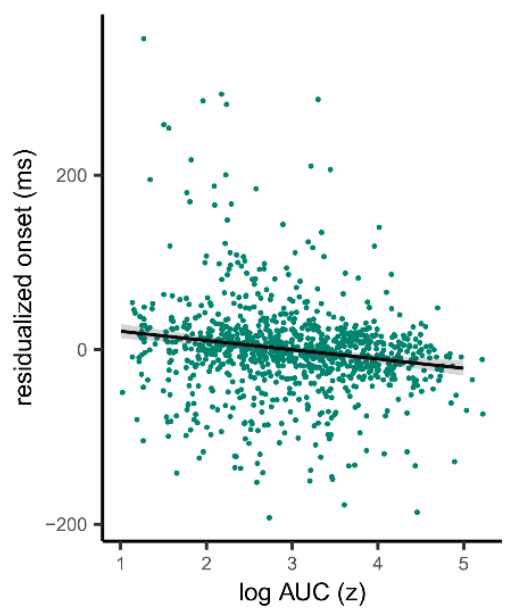

Figure 6. Single trial associations between prEMG onset latency, peak latency, and area under curve (AUC). The dots represent single trials from all participants. The black lines represent the fixed effect model fits from generalized additive mixed models, where the individual variability was modeled as separate intercepts for each participant. The shaded areas represent $95 \%$ confidence intervals.

\section{prEMG variability}

Until now, most previous studies focused on a single estimate of the peak latency of the prEMG. However, prEMG allows for the calculation of the standard deviation of the peak latency for each individual; we can therefore empirically capture the variability of single-trial stopping latencies for each individual (Jana et al., 2020). A caveat here is that that we detect prEMG only in a fraction of the trials, hence the variability estimates are truncated and likely biased. Nonetheless, the prEMG standard deviations show a considerable variability across participants and may therefore be informative of individual differences (Figure 7A). The average individual standard deviation was $40 \mathrm{~ms}$ (group-level sd $=28$ ) and varied from $5 \mathrm{~ms}$ to $143 \mathrm{~ms}$. 
To understand the variability measure better, we first considered whether the between-participants variability could be driven by nuisance factors, such as the number of prEMG trials per person or the amount of baseline noise in the EMG. Secondly, it is possible that the variability of the stopping latency simply reflects variability in general processing, which can simply be assessed through the analysis of go responses without any need for stopping variability measure. Therefore, we correlated the variability of the prEMG peak latency with the variability of the go RTs, where a low correlation would indicate that stopping variability gives information beyond the variability seen in go responses. The correlation matrix is depicted in Figure 7B. Note that we are reporting all correlation coefficients from this exploratory analysis with $p$-values uncorrected for multiple comparisons, as the effect sizes are more informative about potential relationships than their significance.

The individual variability in prEMG peak latency correlated moderately with the number of prEMG trials $(r=0.34, p=0.02)$, but showed weak associations with baseline EMG noise $(r=0.13, p=0.40)$. Neither was the correlation with go RT variability particularly strong $(r=0.18, p=0.24)$. Lastly, we replicated the moderate positive correlation reported by Jana et al. (2020) between the prEMG variability and the variability of the SSRT calculated using the BEESTS-method $(r=0.33, p=0.03)$. The data thus suggest that an individual's standard deviation of the prEMG peak latency provides complementary information on individual differences in stopping behavior.
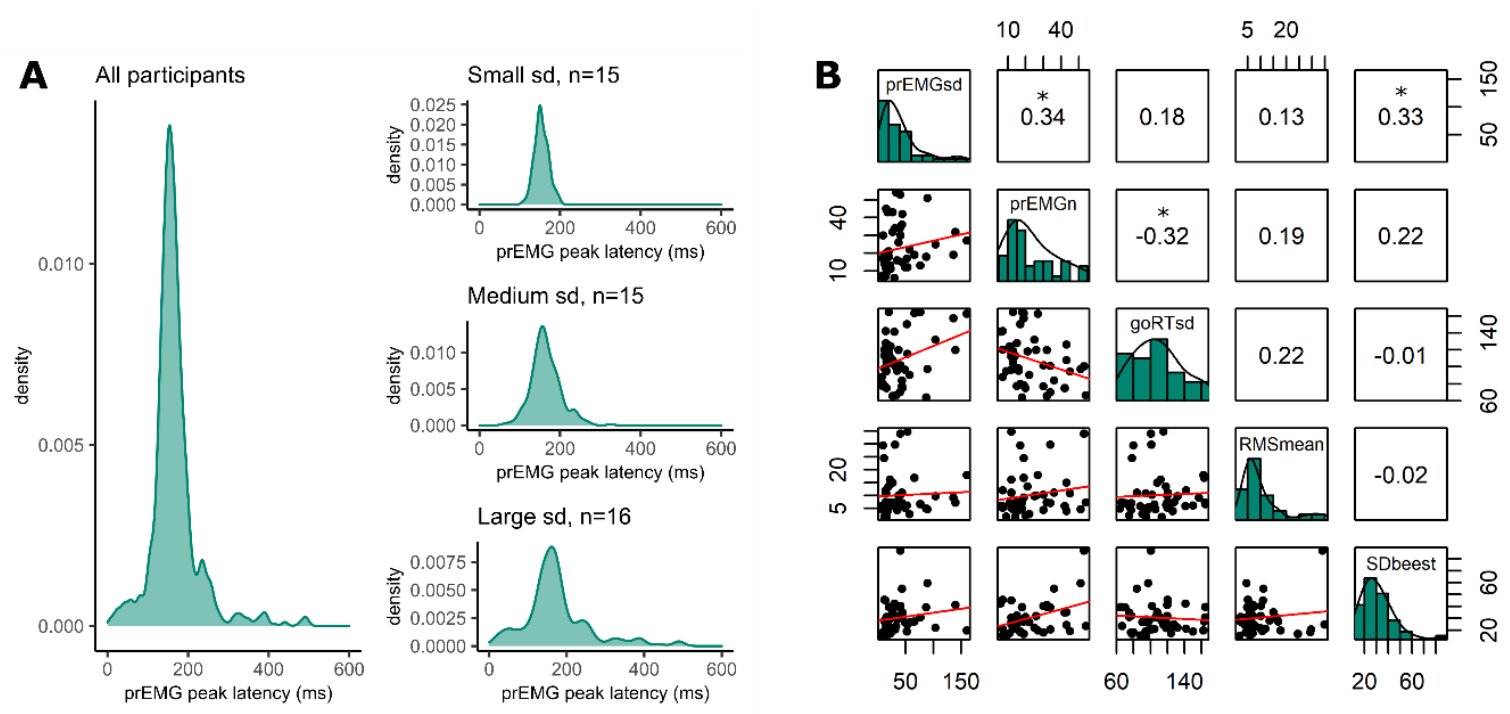

Figure 7. Variability of prEMG peak latency. A. Density functions of prEMG peak latency across all trials and participants (left panel) and separately for group of participants with low, medium, and large individual standard deviations (sd; right panels). B. Correlation matrix between individual prEMG standard deviations (prEMGsd), number of prEMG trials (prEMGn), go reaction time standard deviations (goRTsd), average root mean square EMG amplitudes during the inter-trial-interval -200 to 0 before go stimulus onset (RMSmean), and standard deviations of the SSRT calculated through the BEESTS method (SDbeest). The diagonal shows the histograms for each variable together with the density functions, the lower diagonal shows the scatter plots, and the upper diagonal shows the Spearman correlation coefficients. *marks a significant correlation coefficient at alpha level 0.05 (uncorrected). 


\section{prEMG reliability}

An important question for individual differences research is whether we can reliably estimate the prEMG characteristics despite the varying number of available trials per participant. We therefore estimated the split-half reliability of the prEMG based latency and amplitude measures (Table 4), using a permutation-based split-half reliability approach (Parsons et al., 2019). Using all available trials and participants, we found high reliability for latency measures $(0.94$ and 0.87 for the prEMG onset and peak latency, respectively) but somewhat lower reliability for the prEMG peak amplitude and AUC (0.51 and 0.48 , respectively).

\begin{tabular}{lrrrr} 
& mean & sd & 2.5th & 97.5th \\
\hline onset latency (ms) & 0.94 & 0.01 & 0.91 & 0.97 \\
peak latency (ms) & 0.87 & 0.05 & 0.75 & 0.94 \\
peak RMS amplitude $(\mathrm{z})$ & 0.51 & 0.13 & 0.22 & 0.71 \\
AUC (z) & 0.48 & 0.11 & 0.24 & 0.68 \\
\hline
\end{tabular}

Table 4. Split half reliability estimates for prEMG characteristics, including mean, standard deviation, and percentiles derived from the permutation distributions that correspond to the $95 \%$ confidence intervals. RMS root mean square; AUC - area under the curve from onset to peak.

A critical question for further research is how many trials and participants are necessary for reliable prEMG estimates. For instance, it may seem reasonable to exclude participants with few prEMG trials from further analysis. Yet, this could be harmful for the overall power for the group-level statistics. We repeated the split-half reliability analysis, sampling the lowest number of trials available for all participants and increasing the number of trials while excluding participants if they did not have the necessary number of trials available. This procedure resulted in incremental reliability estimates starting at 46 participants with 6 trials and ending with 12 participants with 32 trials (Figure 8). Interestingly, the reliability estimates for prEMG onset latency remained high (above 0.8 ) no matter the reduction in the number of trials and/or participants. prEMG peak latency reliability increased with the number of trials and reached a plateau around 0.77 after around 13 trials (with increased confidence intervals as the number of participants decreased). As before, the reliability estimates for amplitude measures did not show good reliability, with values hovering around 0.25 and decreasing even further with fewer participants.

In sum, we conclude that prEMG latency measures have overall good reliability, while amplitudebased measures do not. While reliability measures are affected by the specifics of each dataset, our data suggest to base these measures on 15 trials or more. However, the reliability estimates were 
bioRxiv preprint doi: https $/ /$ doi org/10.1101/2021.05.13.443994 this version posted May 14,2021 . The copyright holder for this preprint (which was not certified by peer review) is the author/funder, who has granted bioRxiv a license to display the preprint in perpetuity. It is made available under aCC-BY-NC-ND 4.0 International license.

highest when all participants (even those with less than 10 trials) and all trials where included. Thus, the inclusion of few participants with lower-than-optimal trial numbers may nonetheless be beneficial for group-level statistics.
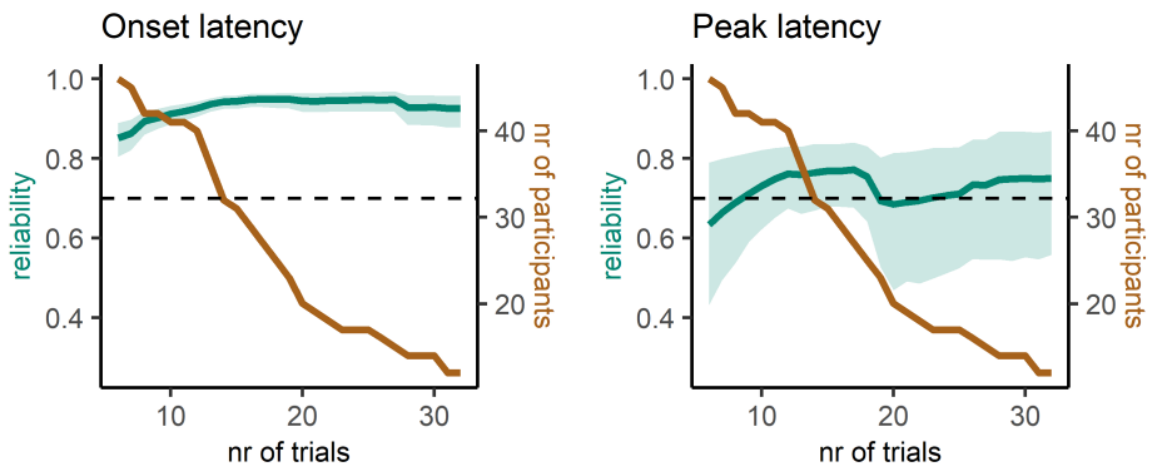

Peak amplitude

AUC (onset to mean)
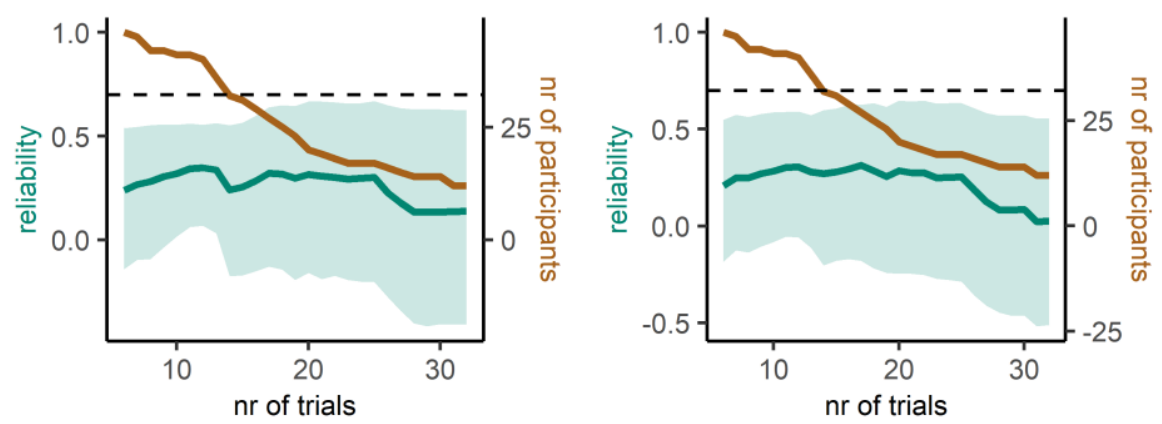

Figure 8. Reliability estimates as a function of number of trials (green) and number of participants (brown) for prEMG latency and amplitude based measures. Green shaded areas represent $95 \%$ confidence intervals for the reliability estimates, and the black dashed horizontal line represent a reliability value of 0.7 for reference. Note the different $y$-axes for latency and amplitude measures. AUC - area under the curve from prEMG onset to peak. 


\section{Box 3. Practical tips for data acquisition}

\section{Recording systems}

EMG can be recorded by dedicated systems, but also by using electroencephalography (EEG) equipment that many human neurophysiology labs already own. In fact, if simultaneous EEG-EMG measures are of interest, it is preferable to record them by the same system so that both signals are synchronized on acquisition. Many EEG systems include a limited number of dedicated channel pairs that allow for the recording of activity referenced against each other instead of referencing against the common EEG reference. Those channels can be used to record from electrodes placed on the skin above muscles to capture EMG. Alternatively, EMG electrodes can be referenced against the common EEG reference during acquisition and then re-referenced offline to get a single bipolar EMG channel. Note that a major caveat of this procedure is that a larger distance between the active EMG electrodes and the EEG reference increases the chance of amplifier saturation, rendering the EMG signal useless.

\section{Movement direction}

The most intuitive movements (for pushing a button, for example) do not necessarily produce the largest signal-to-noise ratio in EMG recordings. We recommend testing various ways of producing a movement before starting the experiment. For thumb muscles (pollicis brevis), the strongest signal is produced by moving the thumb down while keeping the rest of the fingers flat on a surface (it may be necessary to elevate the rest of the hand on a book or a block so that downward thumb movements become possible). For the index finger (first dorsal interosseous), moving the finger horizontally while keeping the palms flat on the table gives the best results. Even though such movements may be unusual for the participants at first, they typically learn fast after some training. Gamified instructions may help to speed up the learning process (e.g. Quoilin et al., 2016).

\section{Avoiding spurious muscle tension}

Even though EMG bursts related to movement are large and clear, detection algorithms may struggle with exact onset and peak latencies if a lot of baseline muscle tension is present. This can be reduced significantly by letting the participants rest their arms comfortably on a pillow, and by reminding them before and during the task to relax their hands. Such verbal reminders typically help even if the participant is not aware of any muscle tension. One potential problem is that specific instructions to relax the hands may interfere with task-related tonic EMG fluctuations, but to the best of our knowledge, such effects have not been investigated systematically. 


\section{Discussion}

We analyzed prEMG from a standard stop signal task to investigate its utility and reliability as a marker of stopping behavior. We compared it thoroughly to the best-established behavioral marker of the stopping latency, the SSRT. Further, we evaluated whether prEMG supports the horse race model that underlies the SSRT estimation in nearly all studies using the stop signal task. We also provided practical tips for EMG data acquisition and analysis (Boxes 2 and 3). We found that prEMG provides unique information about stopping and that assumptions of the horse race model may not regularly be met, questioning the broad and often undifferentiated use of the SSRT as a single indicator of stopping performance. In the following, we elaborate on three main conclusions, which pave the way for outstanding questions about prEMG and further stopping for research.

Conclusion 1: prEMG is partially independent of the SSRT. When comparing the prEMG to the SSRT, there are two main findings: the prEMG peak latency occurs earlier than the SSRT and it correlates moderately-to-highly with the SSRT. It is thus conceivable that the prEMG peak latency and the SSRT capture the same conceptual construct - the individual inhibitory capability - but they simply reflect different stages in the cascade of events. In support of this, Jana et al. (2020) compared the timing of different biomarkers associated with inhibition and proposed a hypothetical timeline of events, with stopping-related changes occurring in the prefrontal cortex at $120 \mathrm{~ms}$, in the motor cortex at $140 \mathrm{~ms}$, at peripheral muscles at $160 \mathrm{~ms}$, and the final suppression of behavior around $220 \mathrm{~ms}$. Accordingly, the $60 \mathrm{~ms}$ delay between the myographic (prEMG peak latency) and the behavioral (SSRT) measures may reflect the final ballistic component of the movement, the so-called point of no return, after which initiated movements are unaffected by any higher-order control mechanisms (Osman et al., 1986). In this case, prEMG may simply reflect the inertia of the neuromuscular system and response device presiding after the prepared movement has been stopped in the cortex (Gopal and Murthy, 2016; Jana et al., 2020). However, such 'leakage' EMG is usually characterized by a gentler onset slope compared with the normal go-responses, and may therefore be differentiated from prEMG bursts where the onset slopes are similar to those of the go-responses (McGarry and Franks, 2003, 1997; see Figure 1B for our data). Further, there is no consensus on what constitutes such a ballistic stage in manual movements or whether it exists at all, as several studies have shown that both cortical and muscle activity can be stopped at any point before their accumulation into a full response (De Jong et al., 1990; Jennings et al., 1992; Scangos and Stuphorn, 2010). Interestingly, the ballistic stage of saccadic movements is well established, but no prEMG can be detected in the monkeys' oculomotor muscles in an antisaccade task (Godlove et al., 2011). 
While the evidence regarding ballistic stage of manual responses may be inconclusive, further results indicate partial dissociation between the prEMG and the SSRT. First, the correlations between the prEMG and SSRT vary considerably between studies from about $10-60 \%$ variance explained, leaving plenty of unexplained variance even for the studies with the highest correlations. Second, the correlational structure with other behavioral and electrophysiological measures derived from the stop signal task differ greatly between the prEMG and the SSRT, where the latter is associated with a speedaccuracy tradeoff between fast going and accurate stopping (Huster et al., 2020). In sum, we conclude that even though prEMG and SSRT seem to share common variance, they are not simply the sequential stages of the same process and thus likely reflect complementary aspects of stopping behavior.

Conclusion 2: prEMG characteristics do not support the independent horse race model. Several results from our analysis indicate incompatibility of the prEMG with the horse race model. On the one hand, we replicated the results from De Jong et al. (1990), who demonstrated that prEMG trials correspond to trials in the go-trial distribution that are fast enough to initiate muscle activity, but slow enough to not outrun the stopping process. On the other hand, neither unsuccessful stop trials nor successful stop trials with prEMG matched the corresponding partitions of the go-EMG distributions. This indicates that the horse race model framework should not be extended to the go-EMG distribution and it is not appropriate for calculating the prEMG-based SSRT. Furthermore, prEMG peak latency varied with the SSD, indicating that stopping latency is not stable, as assumed by the standard horse race model. Lastly, the single trial analysis indicated a major violation of the horse race model independence assumption, as the go process (measured by the go-locked prEMG onset latency) and the stop process (measured by the stop-locked prEMG peak latency) shared about $70 \%$ of variance if the SSD was accounted for. Together, these results add to the increasing pool of evidence that suggests that the independent horse race model is not an appropriate approximation of the stopping performance in practice.

Conclusion 3: prEMG provides unique information about stopping. Having concluded that prEMG is not the same as the SSRT, we propose that the prEMG is a useful measure on its own. One of its main advantages is that it is not a summary estimate like the SSRT, but provides single-trial stopping latencies as well as an empirical stopping variability estimate for each individual. In our analysis, we found that participants differed in the variability of prEMG peak latencies and that this variability was not driven by nuisance factors, being therefore potentially meaningful for investigating individual differences. Further, we found that prEMG peak latency varied with the SSD, but did not vary across the course of the experiment, providing a quantifiable test for a previously elusive question about whether there are short-term practice effects of stopping. Lastly, the reliability estimates for the prEMG latency measures were above 0.7 even with relatively few trials per participant. As such, we 
advocate using prEMG as a standard outcome variable in the stop signal task, as it provides unique, useful, and reliable information about stopping behavior.

Outstanding question 1: Is prEMG peak latency a marker of individual inhibition capacity? While prEMG can be considered an assumption-free physiological measure in its implementation, it is not free from assumptions in its interpretation. The most urgent unresolved question is whether prEMG captures the latency of inhibitory mechanisms in the brain. On the one hand, prEMG decline unequivocally indicates the stopping of muscle activity, so it is safe to presume that the peak latency represents the latency of the stopping of behavior. On the other hand, the actual mechanisms that cause this EMG decline cannot be derived from prEMG alone. Several studies have investigated whether stopping of specific muscle activity could be caused by the activation of antagonist muscles and found that agonist-stopping occurs before antagonist-activation; thus, the former cannot be caused by the latter (Atsma et al., 2018; Corneil et al., 2013; Goonetilleke et al., 2012, 2010; Scangos and Stuphorn, 2010). However, it remains to be seen whether prEMG declines because of a reduction in response maintenance (for example by restoring the inhibitory state of the cortical-basal gangliathalamic loop during rest), or because of an active inhibition mechanism that blocks ongoing motor activity (e.g. by blocking the direct pathway output by increased inhibition via the sub-thalamic nucleus, or by direct inhibitory influences on M1; e.g. Hynd et al., 2021). Complementary simultaneous data from brain imaging and stimulation are necessary to understand the neural mechanisms that drive the halt in muscle activity.

Outstanding question 2. Are prEMG trials representative for all successful stop trials? Even though prEMG can provide single-trial estimates for each participant and is therefore more informative than the SSRT, it is still expressed in only a fraction of successful stop trials. Could these trials be qualitatively different from other successful stop trials? McGarry and Franks (1997) designed a stop signal task with untypically short SSDs, and found that, based on the shape of the prEMG rising slope, trials could be divided into trials with early and late stopping mechanisms (see Box 1 about interrupted response for details). Further, electroencephalography studies have found that prEMG trials show decreased lateralized readiness potentials and increased frontal negativities (De Jong et al., 1990; van Boxtel et al., 2001). Increased frontal negativities have also been related to awareness of the prEMG in response conflict tasks (Ficarella et al., 2019). However, whether these cortical changes reflect qualitatively different mechanisms or merely a continuous variation in the strength of the underlying processes is unresolved (van Boxtel et al., 2001).

Outstanding question 3: Are the mechanisms driving prEMG ubiquitous across contexts? While it has been shown that the SSRT becomes increasingly unreliable in more complex stop signal tasks and its 
use is thus discouraged (Bissett and Logan, 2014), prEMG can nonetheless still be recorded in such task settings (Raud et al., 2020a; Raud and Huster, 2017). Further, (pr)EMG is not limited to the stop signal task, but can be recorded in any task that requires overt responses, as well as tasks that require more complex movements activating several muscle groups (Tao et al., 2018). As such, EMG can provide highly sought-after physiological measures that are comparable across different contexts. We argue that this is most useful approach for the study of response inhibition in tasks that rely on the absence of overt behavior as the primary outcome measure. For example, by comparing inhibitory mechanisms across different tasks, it was found that the prEMG peaked significantly later in the go/nogo task than in the stop signal task, which was also paralleled by differences in the cortical activity, thus suggesting that these tasks may recruit different inhibitory mechanisms (Raud et al., 2020b). Comparisons of prEMG characteristics and their driving mechanisms in different contexts provide a rich ground for future research on motor and cognitive control.

Conclusions. Based on the synthesis of previous work together with a comprehensive analysis of prEMG characteristics in an independent dataset, we conclude that prEMG is a unique and reliable measure of stopping. It should be considered a separate indicator from the SSRT, as it does not support the assumptions of the horse race model. PrEMG is not limited to specific muscles, stimulus modalities, or task contexts, so it provides a comparative physiological measure for a range of different scenarios. Therefore, we encourage the widespread use of prEMG to investigate the underlying mechanisms of response inhibition, as well as individual differences in healthy and clinical samples. 


\section{Materials and methods}

\section{Sample}

Data was analyzed from 46 healthy young adults (mean age $=23.7, s d=5.5,30$ females, 3 left-handed). Initially, data was collected from 52 participants, but four were discarded due to technical issues with the response device and another two were discarded as statistical outliers (above two inter-quartile ranges) based on EMG onset and peak latencies. Participants gave written informed consent prior to data collection, and everyone received monetary compensation for their participation. The study was conducted in accordance with the Helsinki declaration, and was approved by the internal review board of the University of Oslo. The data and analyses scripts are available in an accompanying project in the Open Science Framework (Raud et al., 2021).

\section{Stop signal task and behavioral variables}

All participants performed a choice-reaction time task and a stop signal task presented in alternating blocks (six blocks in total). Task presentation was controlled by E-prime 2.0 (Psychology Software Tools, Pittsburgh, PA). For this analysis, we focus on the stop signal task exclusively. The primary task was to respond with a button press using the thumb of the left or right hand to an arrow pointing to the left or right, correspondingly. In $24 \%$ of the trials, a stop signal appeared after a variable stop signal delay, instructing participants to stop their initiated response. In total, there were 684 go trials and 216 stop trials, equally distributed between left- and right-hand trials.

Each trial started with a black fixation cross (duration jittered between 500-1000 ms), followed by a colored arrow as a go signal, presented for $100 \mathrm{~ms}$. In stop trials, a second arrow of a different color was presented after the SSD. The SSD could vary between 100-800 ms and was adjusted based on an adaptive tracking procedure collapsed across both hands: The initial SSD was set to $250 \mathrm{~ms}$, and then increased or decreased by $50 \mathrm{~ms}$ following successful and unsuccessful stop trials, respectively. Responses were collected up to $1000 \mathrm{~ms}$ after the go stimulus and potential responses after this window were treated as omissions. All stimuli were presented centrally against a grey background and the stimulus color assignments (green, orange, or blue) were counterbalanced across participants. Trial order within a block was randomized, except for the first block, which always started with a minimum of 10 go trials. Short pauses ( 9 seconds) were given after every 75 trials, and a longer break with a duration of roughly 5 minutes was allowed halfway through the task.

Prior to the experiment, the participants completed a short training block (20 trials, $50 \%$ stop trials) with computerized trial-by-trial feedback. In addition, participants were told that it was important to 
be both fast and accurate, that they were not supposed to wait for the stop-signal, and that it was not possible to successfully stop in all stop trials. Automated feedback was presented on screen after every 75th trial. Here, participants were instructed to be faster if their average goRT was above $600 \mathrm{~ms}$, and to be more accurate if the average stop accuracy was below $40 \%$. If neither of these applied, they received the feedback 'Well done!'.

The following behavioral variables were extracted based on task performance: go accuracies, error, and omission rates, go reaction times, stop accuracies, unsuccessful stop reaction times, average stop signal delay, and SSRTs. These were calculated using the integration method, where corresponding go reaction time distributions included choice errors, and omissions were replaced by the maximum reaction time (Verbruggen et al., 2019).

We also estimated SSRTs and their variability using a Bayesian parametric approach (BEESTS; Matzke et al., 2019, 2013). We chose the BEESTS2-GF model due to the low amount of choice errors in our sample. This model assumes a single go process as well as a stop process, and that both the go and stop signal reaction times can be approximated by an ex-Gaussian distribution, defined by the $\mu$ and $\tau$ parameters. As this model only fits one go process, choice errors were removed prior to analysis. We also removed improbable and outlier trials, defined as trials with RTs below $100 \mathrm{~ms}$ and/or RTs $-/+3.5$ SDs away from each participant's mean. Estimation was run using the Dynamic Models of Choice toolbox (Heathcote et al., 2019) in R Studio (version 1.3.1093) where models were fitted to each participant individually. Parameter priors were defined as weakly informative truncated normal distributions (Heathcote et al., 2019; Skippen et al., 2019). Specifically, we set the prior means to 500 $\mathrm{ms}$ for $\mu_{\mathrm{go}}$ and $\mu_{\mathrm{stop}}, 200 \mathrm{~ms}$ for $\sigma_{\mathrm{go}}$ and $\tau_{\mathrm{go}}, 100 \mathrm{~ms}$ for $\sigma_{\mathrm{stop}}$ and $\tau_{\mathrm{stop}}$, and $1000 \mathrm{~ms}$ for the standard deviations. The priors for go and trigger failures had a mean of 1.5 (probit scale) with a standard deviation of 1 . We ran 3 chains for each estimated parameter, and thus a total of 24 chains. All rhatvalues were $<1.1$, indicating chain convergence. Posterior distributions were updated well, suggesting that priors were largely uninformative. Plots showing the prior relative to posterior distributions, chains and posterior predictive model checks can be found in the accompanying materials on OSF (Raud et al., 2021).

\section{EMG acquisition}

Two surface EMG electrodes were placed on the skin above the abductor pollicis brevis in parallel to the belly of the muscles on each hand using bipolar $\mathrm{Ag} / \mathrm{Ag}-\mathrm{Cl}$ montages. Corresponding ground electrodes were placed on each forearm. The recordings were performed using a BrainAmp ExG 
extension for bipolar recordings (Brain Products $\mathrm{GmbH}$, Germany) with an online low-pass filter of $1000 \mathrm{~Hz}$, a sampling rate of $5000 \mathrm{~Hz}$, and a $0.5 \mu \mathrm{V}$ resolution.

\section{EMG preprocessing and burst detection}

EMG preprocessing and variable extractions were performed with custom scripts in MATLAB (The Math Works, Inc. MATLAB, version 2018a). The EMG signal was bandpass filtered between $20-250 \mathrm{~Hz}$ using a second-order butterworth filter and resampled to $500 \mathrm{~Hz}$. Data epochs were extracted for all trials from -0.2 to $1.6 \mathrm{sec}$ relative to go signal onset. Trials were rejected at this stage if the average baseline activity was larger than $100 \mu \mathrm{V}$ (across all participants, this led to the rejection of 206 trials, i.e. roughly $0.5 \%)$.

The data epochs were transformed by taking the root mean square over a moving average window or +/-5 datapoints, and normalized to baseline by dividing the full time-course by the average of the baseline period ( -0.2 to 0 sec relative to the go signal). For each participant and each hand, epochs were then concatenated and z-scored block-wise.

EMG bursts were identified in a trial if any data point exceeded a threshold of 1.2 (in standard deviation units, taking into account all trials). This threshold was confirmed visually and has been held constant across several recent studies (Huster et al., 2020; Raud et al., 2020a, 2020b; Thunberg et al., 2020). The peak latency was defined as the time-point of the highest amplitude in a trial. Onset latency was determined by tracking amplitude values backwards in time, starting at the peak latency, up until continuous datapoints of $8 \mathrm{~ms}$ were below amplitude-threshold. Lastly, peak latencies of the EMG in stop trials were re-calculated relative to the stop signal onset by subtracting the stop signal delay. All variables were averaged over left- and right- hand trials.

\section{Statistical analyses}

Statistical analyses were performed using $R$ ( $R$ Core Team, 2019) and the package collection of tidyverse (Wickham et al., 2019) and custom scripts in MATLAB. All figures were created either in MATLAB or in R using the packages ggplot2 (Wickham et al., 2020, p. 2), ggpubr (Kassambara, 2020), and psych (Revelle, 2021).

The trial differences between EMG amplitudes and peaks were tested by means of $t$-tests and ANOVAs within the Bayesian framework using the R package BayesFactor (Morey et al., 2018). The evidence was quantified using the Bayes factor, with values greater than 1 indicating support for the alternative 
and values below 1 for the null hypothesis (with $>3$ and $<0.3$ often considered indicators of strong evidence towards alternative or null, respectively).

We further tested whether the prEMG characteristics (inhibition functions, probabilities, onset latencies, peak latencies, peak amplitudes) varied across SSDs. To account for individual variability in SSDs, we centralized the SSDs per participant instead of using the absolute values. The mode of each participant's SSD distribution was treated as zero and we extracted the values +/-3 SSD steps around the mode. The prEMG characteristics were averaged per SSD, so that each participant had one value per SSD, given that they had any trials with prEMG at that specific SSD.

The relationships between the prEMG characteristics and the prEMG were expected to be non-linear with the exact shape unknown. Therefore, these were tested by the general additive mixed models (GAMM) using the R package gamm4 (Wood and Scheipl, 2020). GAMM includes the possibility of adding a smooth term to the model, which is a linear sum of several splines. The degree of nonlinearity (or wiggliness) of the resulting model fit is determined by the estimated degrees of freedom (edf) and the corresponding $\mathrm{F}$ and $\mathrm{p}$-values, together with the estimated lambda factor that penalizes for overfitting. For example, in cases where linear fit is the best fit, the edf will be close to one; in case of second-order polynomial fit, the edf will be close to two, etc. We considered smooth parameters with a p-value below 0.05 as indicative of a significant effect.

Single trial analysis. The single trial analysis was done on prEMG (successful stop trials) only, using prEMG onset latencies, peak latencies, and areas under the curve (AUC) from onset to peaks. These were calculated as a cumulative sum of the amplitudes from onset to peak. AUC thus integrates the duration and amplitude of the prEMG bursts before their decline. As the bivariate correlations with the SSD at a single trial level were high for the onset latencies $(r=0.88)$, they were orthogonalized for the SSD by predicting onsets from the SSD for each participant and using the residuals instead of original values. AUCs were log-transformed since the original values were extremely skewed towards low values. Additional data cleaning was applied, in which trials were excluded if the peak latency occurred before the stop signal or if the onsets (go-locked) or peaks (stop-locked) exceeded two interquartile ranges of the individual participant's data. Three separate GAMMs were performed predicting peak latency from onset latency, AUC from peak latency, and AUC from onset latency.

Variability. Individual prEMG variability was estimated as the standard deviation of the prEMG peak latencies across trials for each participant. Bivariate Pearson rank-ordered correlation coefficients and (uncorrected) $p$-values were used to identify relationships between prEMG variability and the number of prEMG trials per participant, go reaction time standard deviations, average root mean square 
amplitudes during the inter-trial-interval of -20 to $0 \mathrm{~ms}$ before go stimulus, and standard deviation of the SSRT calculated by the BEESTS method.

Reliability. We estimated the internal consistency of the different prEMG metrics (onset latencies, peak latencies, peak amplitudes, and AUCs from onset to peak) using a permutation-based split-half approach (Parsons et al., 2019). After the same level of additional data cleaning as in the single trial analyses, we split the data for each participant into two random halves and calculated the mean of each half. These estimates for each half were then correlated across participants and the correlations were corrected using the Spearman-Brown prophecy formula (Brown, 1910; Spearman, 1910). This process was repeated 10000 times, thus resulting in a distribution of reliability estimates. We used the mean of this distribution as our summary reliability estimate, and $2.5^{\text {th }}$ and $97.5^{\text {th }}$ percentiles as the upper and lower limits of the confidence intervals.

Initially, all trials from all participants were used for the reliability estimates. Next, we repeated the analysis iteratively using an equal number of trials per participant and excluding all participants who did not have enough trials available for a specific iteration. We started with 6 trials per participant (lowest number of trials available for all participants) and increased the number of trials per iteration by one until we were left with 32 trials per participant (with 12 participants remaining). We repeated this process 1000 times, thus getting a distribution of reliability estimates at each trial count.

\section{Acknowledgments}

We would like to thank Celina Müller, Sandra Klonteig, Josefine Bergseth, Thea Wiker, and Karl Zimmerman for their help with data collection.

\section{Competing interests}

The authors declare no competing interests. 


\section{References}

Atsma J, Maij F, Gu C, Medendorp WP, Corneil BD. 2018. Active Braking of Whole-Arm Reaching Movements Provides Single-Trial Neuromuscular Measures of Movement Cancellation. J Neurosci 38:4367-4382. doi:10.1523/JNEUROSCI.1745-17.2018

Band GPH, van der Molen MW, Logan GD. 2003. Horse-race model simulations of the stop-signal procedure. Acta Psychol (Amst) 112:105-142.

Best M, Lawrence NS, Logan GD, McLaren IPL, Verbruggen F. 2016. Should I Stop or Should I Go? The Role of Associations and Expectancies. J Exp Psychol Hum Percept Perform 42:115-137. doi:10.1037/xhp0000116

Bissett PG, Hagen MP, Jones HM, Poldrack RA. 2021a. Design issues and solutions for stop-signal data from the Adolescent Brain Cognitive Development (ABCD) study. Elife 10. doi:10.7554/eLife.60185

Bissett PG, Jones HM, Poldrack RA, Logan GD. 2021b. Severe violations of independence in response inhibition tasks. Science Advances 7:eabf4355. doi:10.1126/sciadv.abf4355

Bissett PG, Logan GD. 2014. Selective stopping? Maybe not. J Exp Psychol Gen 143:455-472. doi:10.1037/a0032122

Boucher L, Palmeri TJ, Logan GD, Schall JD. 2007. Inhibitory control in mind and brain: an interactive race model of countermanding saccades. Psychol Rev 114:376-397. doi:10.1037/0033295X.114.2.376

Brown W. 1910. Some Experimental Results in the Correlation of Mental Abilities1. British Journal of Psychology, 1904-1920 3:296-322. doi:https://doi.org/10.1111/j.2044-8295.1910.tb00207.x

Burle B, Possamaï C-A, Vidal F, Bonnet M, Hasbroucq T. 2002. Executive control in the Simon effect: an electromyographic and distributional analysis. Psychological Research 66:324-336. doi:10.1007/s00426-002-0105-6

Coles MGH, Scheffers MK, Fournier L. 1995. Where did you go wrong? Errors, partial errors, and the nature of human information processing. Acta Psychologica, Discrete and Continuous Information Processing 90:129-144. doi:10.1016/0001-6918(95)00020-U

Corneil BD, Cheng JC, Goonetilleke SC. 2013. Dynamic and opposing adjustment of movement cancellation and generation in an oculomotor countermanding task. J Neurosci 33:99759984. doi:10.1523/JNEUROSCI.2543-12.2013

Coxon JP, Stinear CM, Byblow WD. 2006. Intracortical inhibition during volitional inhibition of prepared action. J Neurophysio/ 95:3371-3383. doi:10.1152/jn.01334.2005

De Jong R, Coles MG, Logan GD, Gratton G. 1990. In search of the point of no return: the control of response processes. J Exp Psychol Hum Percept Perform 16:164-182.

Ficarella SC, Rochet N, Burle B. 2019. Becoming aware of subliminal responses: An EEG/EMG study on partial error detection and correction in humans. Cortex 120:443-456. doi:10.1016/j.cortex.2019.07.007

Godlove DC, Garr AK, Woodman GF, Schall JD. 2011. Measurement of the extraocular spike potential during saccade countermanding. Journal of Neurophysiology 106:104-114. doi:10.1152/jn.00896.2010

Goonetilleke SC, Doherty TJ, Corneil BD. 2010. A within-trial measure of the stop signal reaction time in a head-unrestrained oculomotor countermanding task. J Neurophysiol 104:3677-3690. doi:10.1152/jn.00495.2010

Goonetilleke SC, Wong JP, Corneil BD. 2012. Validation of a within-trial measure of the oculomotor stop process. Journal of Neurophysiology 108:760-770. doi:10.1152/jn.00174.2012

Gopal A, Murthy A. 2016. A common control signal and a ballistic stage can explain the control of coordinated eye-hand movements. Journal of Neurophysiology 115:2470-2484.

doi:10.1152/jn.00910.2015 
Hannah R, Muralidharan V, Sundby KK, Aron AR. 2020. Temporally-precise disruption of prefrontal cortex informed by the timing of beta bursts impairs human action-stopping. Neurolmage 222:117222. doi:10.1016/j.neuroimage.2020.117222

Heathcote A, Lin Y-S, Reynolds A, Strickland L, Gretton M, Matzke D. 2019. Dynamic models of choice. Behav Res Methods 51:961-985. doi:10.3758/s13428-018-1067-y

Huster RJ, Messel MS, Thunberg C, Raud L. 2020. The P300 as marker of inhibitory control - Fact or fiction? Cortex 132:334-348. doi:10.1016/j.cortex.2020.05.021

Hynd M, Soh C, Rangel BO, Wessel JR. 2021. Paired-pulse TMS and scalp EEG reveal systematic relationship between inhibitory GABAa signaling in $\mathrm{M} 1$ and fronto-central cortical activity during action stopping. J Neurophysiol 125:648-660. doi:10.1152/jn.00571.2020

Jackson N, Greenhouse I. 2019. VETA: An Open-Source Matlab-Based Toolbox for the Collection and Analysis of Electromyography Combined With Transcranial Magnetic Stimulation. Front Neurosci 13. doi:10.3389/fnins.2019.00975

Jahanshahi M, Obeso I, Baunez C, Alegre M, Krack P. 2015. Parkinson's disease, the subthalamic nucleus, inhibition, and impulsivity. Mov Disord 30:128-140. doi:10.1002/mds.26049

Jana S, Hannah R, Muralidharan V, Aron AR. 2020. Temporal cascade of frontal, motor and muscle processes underlying human action-stopping. eLife 9:e50371. doi:10.7554/eLife.50371

Jennings JR, Van der Molen MW, Brock K, Somsen RJ. 1992. On the synchrony of stopping motor responses and delaying heartbeats. Journal of Experimental Psychology: Human Perception and Performance 18:422-436. doi:10.1037/0096-1523.18.2.422

Kassambara A. 2020. ggpubr: "ggplot2" Based Publication Ready Plots.

Lipszyc J, Schachar R. 2010. Inhibitory control and psychopathology: a meta-analysis of studies using the stop signal task. J Int Neuropsychol Soc 16:1064-1076. doi:10.1017/S1355617710000895

Liu J, Ying D, Rymer WZ, Zhou P. 2015. Robust muscle activity onset detection using an unsupervised electromyogram learning framework. PLoS One 10:e0127990. doi:10.1371/journal.pone.0127990

Logan GD, Cowan WB. 1984. On the ability to inhibit thought and action: A theory of an act of control. Psychological Review 91:295-327. doi:10.1037/0033-295X.91.3.295

Matzke D, Curley S, Gong CQ, Heathcote A. 2019. Inhibiting responses to difficult choices. J Exp Psychol Gen 148:124-142. doi:10.1037/xge0000525

Matzke D, Love J, Heathcote A. 2017. A Bayesian approach for estimating the probability of trigger failures in the stop-signal paradigm. Behav Res Methods 49:267-281. doi:10.3758/s13428015-0695-8

Matzke D, Love J, Wiecki TV, Brown SD, Logan GD, Wagenmakers E-J. 2013. Release the BEESTS: Bayesian Estimation of Ex-Gaussian STop-Signal reaction time distributions. Front Psychol 4:918. doi:10.3389/fpsyg.2013.00918

McGarry T, Franks IM. 2003. On the nature of stopping an earlier intended voluntary action. Motor Control 7:155-198. doi:10.1123/mcj.7.2.155

McGarry T, Franks IM. 1997. A horse race between independent processes: evidence for a phantom point of no return in preparation of a speeded motor response. J Exp Psychol Hum Percept Perform 23:1533-1542.

Morey RD, Rouder JN, Jamil T, Urbanek S, Forner K, Ly A. 2018. BayesFactor: Computation of Bayes Factors for Common Designs.

Osman A, Kornblum S, Meyer DE. 1986. The point of no return in choice reaction time: controlled and ballistic stages of response preparation. J Exp Psychol Hum Percept Perform 12:243-258. doi:10.1037//0096-1523.12.3.243

Parsons S, Kruijt A-W, Fox E. 2019. Psychological Science Needs a Standard Practice of Reporting the Reliability of Cognitive-Behavioral Measurements. Advances in Methods and Practices in Psychological Science 2:378-395. doi:10.1177/2515245919879695 
Quoilin C, Lambert J, Jacob B, Klein P-A, Duque J. 2016. Comparison of Motor Inhibition in Variants of the Instructed-Delay Choice Reaction Time Task. PLOS ONE 11:e0161964. doi:10.1371/journal.pone.0161964

Raud L, Huster RJ. 2017. The Temporal Dynamics of Response Inhibition and their Modulation by Cognitive Control. Brain Topogr 30:486-501. doi:10.1007/s10548-017-0566-y

Raud L, Huster RJ, Ivry RB, Labruna L, Messel MS, Greenhouse I. 2020a. A Single Mechanism for Global and Selective Response Inhibition under the Influence of Motor Preparation. J Neurosci 40:7921-7935. doi:10.1523/JNEUROSCI.0607-20.2020

Raud L, Thunberg C, Huster R. 2021. Partial response electromyography as a marker of the individual stopping latency. Data and analyses scripts. doi:10.17605/OSF.IO/RQNUJ

Raud L, Westerhausen R, Dooley N, Huster RJ. 2020b. Differences in unity: The go/no-go and stop signal tasks rely on different mechanisms. Neuroimage 210:116582.

doi:10.1016/j.neuroimage.2020.116582

Revelle W. 2021. psych: Procedures for Psychological, Psychometric, and Personality Research.

Rochet N, Spieser L, Casini L, Hasbroucq T, Burle B. 2014. Detecting and correcting partial errors: Evidence for efficient control without conscious access. Cogn Affect Behav Neurosci 14:970982. doi:10.3758/s13415-013-0232-0

Scangos KW, Stuphorn V. 2010. Medial frontal cortex motivates but does not control movement initiation in the countermanding task. J Neurosci 30:1968-1982. doi:10.1523/JNEUROSCI.4509-09.2010

Skippen P, Fulham WR, Michie PT, Matzke D, Heathcote A, Karayanidis F. 2020. Reconsidering electrophysiological markers of response inhibition in light of trigger failures in the stopsignal task. Psychophysiology 57:e13619. doi:https://doi.org/10.1111/psyp.13619

Skippen P, Matzke D, Heathcote A, Fulham WR, Michie P, Karayanidis F. 2019. Reliability of triggering inhibitory process is a better predictor of impulsivity than SSRT. Acta Psychologica 192:104117. doi:10.1016/j.actpsy.2018.10.016

Smith JL, Mattick RP, Jamadar SD, Iredale JM. 2014. Deficits in behavioural inhibition in substance abuse and addiction: a meta-analysis. Drug Alcohol Depend 145:1-33. doi:10.1016/j.drugalcdep.2014.08.009

Snyder HR, Miyake A, Hankin BL. 2015. Advancing understanding of executive function impairments and psychopathology: bridging the gap between clinical and cognitive approaches. Front Psychol 6:328. doi:10.3389/fpsyg.2015.00328

Spearman C. 1910. Correlation Calculated from Faulty Data. British Journal of Psychology, 1904-1920 3:271-295. doi:https://doi.org/10.1111/j.2044-8295.1910.tb00206.x

Sundby KK, Jana S, Aron AR. 2020. Double-blind disruption of right inferior frontal cortex with TMS reduces right frontal beta power for action stopping. Journal of Neurophysiology 125:140153. doi:10.1152/jn.00459.2020

Tao G, Khan AZ, Blohm G. 2018. Corrective response times in a coordinated eye-head-arm countermanding task. J Neurophysiol 119:2036-2051. doi:10.1152/jn.00460.2017

Tatz JR, Soh C, Wessel JR. 2021. Towards a two-stage model of action-stopping: Attentional capture explains motor inhibition during early stop-signal processing. bioRxiv 2021.02.26.433098. doi:10.1101/2021.02.26.433098

Thunberg C, Messel MS, Raud L, Huster RJ. 2020. tDCS over the inferior frontal gyri and visual cortices did not improve response inhibition. Scientific Reports 10:7749. doi:10.1038/s41598-020-62921-z

van Boxtel GJ, van der Molen MW, Jennings JR, Brunia CH. 2001. A psychophysiological analysis of inhibitory motor control in the stop-signal paradigm. Biol Psychol 58:229-262.

van de Laar MC, van den Wildenberg WPM, van Boxtel GJM, van der Molen MW. 2014.

Development of response activation and inhibition in a selective stop-signal task. Biol Psychol 102:54-67. doi:10.1016/j.biopsycho.2014.06.003 
bioRxiv preprint doi: https://doi.org/10.1101/2021.05.13.443994 - this version posted May 14,2021 . The copyright holder for this preprint (which was not certified by peer review) is the author/funder, who has granted bioRxiv a license to display the preprint in perpetuity. It is made available under aCC-BY-NC-ND 4.0 International license.

Verbruggen F, Aron AR, Band GP, Beste C, Bissett PG, Brockett AT, Brown JW, Chamberlain SR, Chambers CD, Colonius H, Colzato LS, Corneil BD, Coxon JP, Dupuis A, Eagle DM, Garavan H, Greenhouse I, Heathcote A, Huster RJ, Jahfari S, Kenemans JL, Leunissen I, Li C-SR, Logan GD, Matzke D, Morein-Zamir S, Murthy A, Paré M, Poldrack RA, Ridderinkhof KR, Robbins TW, Roesch M, Rubia K, Schachar RJ, Schall JD, Stock A-K, Swann NC, Thakkar KN, van der Molen MW, Vermeylen L, Vink M, Wessel JR, Whelan R, Zandbelt BB, Boehler CN. 2019. A consensus guide to capturing the ability to inhibit actions and impulsive behaviors in the stop-signal task. elife 8:e46323. doi:10.7554/eLife.46323

Verbruggen F, Best M, Bowditch WA, Stevens T, McLaren IPL. 2014. The inhibitory control reflex. Neuropsychologia 65:263-278. doi:10.1016/j.neuropsychologia.2014.08.014

Verbruggen F, Chambers CD, Logan GD. 2013. Fictitious inhibitory differences: how skewness and slowing distort the estimation of stopping latencies. Psychol Sci 24:352-362. doi:10.1177/0956797612457390

Verbruggen F, Logan GD. 2008. Automatic and controlled response inhibition: associative learning in the go/no-go and stop-signal paradigms. J Exp Psychol Gen 137:649-672. doi:10.1037/a0013170

Wickham H, Averick M, Bryan J, Chang W, McGowan LD, François R, Grolemund G, Hayes A, Henry L, Hester J, Kuhn M, Pedersen TL, Miller E, Bache SM, Müller K, Ooms J, Robinson D, Seidel DP, Spinu V, Takahashi K, Vaughan D, Wilke C, Woo K, Yutani H. 2019. Welcome to the Tidyverse. Journal of Open Source Software 4:1686. doi:10.21105/joss.01686

Wickham H, Chang W, Henry L, Pedersen TL, Takahashi K, Wilke C, Woo K, Yutani H, Dunnington D, RStudio. 2020. ggplot2: Create Elegant Data Visualisations Using the Grammar of Graphics.

Wood S, Scheipl F. 2020. gamm4: Generalized Additive Mixed Models using "mgcv" and "Ime4."

Wylie SA, Claassen DO, Kanoff KE, Ridderinkhof KR, van den Wildenberg WPM. 2013. Impaired inhibition of prepotent motor actions in patients with Tourette syndrome. J Psychiatry Neurosci 38:349-356. doi:10.1503/jpn.120138

Yaniv A, Benaroya-Milshtein N, Steinberg T, Ruhrrman D, Apter A, Lavidor M. 2017. Specific executive control impairments in Tourette syndrome: The role of response inhibition. Res Dev Disabil 61:1-10. doi:10.1016/j.ridd.2016.12.007 